\title{
Estimations de la résolvante pour une molécule diatomique dans l'approximation de Born-Oppenheimer.
}

\author{
Th.Jecko ${ }^{1}$ \\ Fachbereich Mathematik MA 7-2, \\ Technische Universität Berlin, \\ D-10623 Berlin, Germany, \\ e-mail : jecko@math.tu-berlin.de
}

\begin{abstract}
Résumé
Making use of an adiabatic operator, that takes several electronic states into account, we derive a Born-Oppenheimer approximation of the resolvent for a diatomic molecule. This is an improvement of a result in [KMW1]. Such a resolvent approximation is useful to obtain an adiabatic approximation of total cross-sections (see [Jec2]). The strategy we use, based on Mourre's commutator method and on a new kind of global escape function, may be carried over to control the resolvent of some matricial Schrödinger operators. In the same way, we obtain a semiclassical estimate for the resolvent of the semiclassical Dirac operator with scalar electric potential, extending a result of $[\mathrm{Ce}]$.
\end{abstract}

\section{Introduction.}

En 1927, M.Born et R.Oppenheimer introduisaient ce qui allait devenir l'approximation de Born-Oppenheimer (cf. [BO]), une approche essentielle pour la chimie moléculaire. En vue de décrire les niveaux d'énergie moléculaires, leur idée consistait à profiter du fait que les rapports de la masse de l'électron à celles des noyaux sont très petits, pour effectuer des développements en puissances de $h$, un petit paramètre lié à ces rapports. En théorie de la diffusion, on peut reprendre cette idée, se ramener à une étude semi-classique et profiter ainsi des développements récents dans ce domaine.

Le présent travail est motivé par l'étude de l'approximation de Born-Oppenheimer de sections efficaces totales pour une molécule diatomique (cf. [Jec2]). Afin de pouvoir considérer des processus de diffusion inélastiques, on améliore ici l'approximation de la résolvante obtenue dans [KMW1]. Au moyen d'un opérateur adiabatique, qui prend en compte plusieurs états électroniques et sous une hypothèse de "non-croisement" des niveaux électroniques correspondants, on établit la même approximation de la résolvante que dans [KMW1],

\footnotetext{
${ }^{1}$ previous address : Département de mathématiques, Université de Nantes, 44072 Nantes cedex 03, France, e-mail : jecko@math.univ-nantes.fr
} 
dans une situation plus générale. Comme dans [KMW1], on en déduit une approximation adiabatique pour certains opérateurs d'onde de canal.

Ces estimations semi-classiques de résolvantes sont valables près d'une énergie non-captive pour certains hamiltoniens classiques, qui ne sont pas, comme dans [W1], les sous-hamiltoniens du système à $N$-corps. Elles sont obtenues par la méthode de Mourre (cf. [Mo]).

A la différence des estimations de résolvantes (cf. [PSS]), utilisées dans [Ra] pour l'étude d'opérateurs d'onde et dans [CT] pour celle de l'amplitude de diffusion, les poids, intervenant dans les présentes estimations, ne contiennent pas toutes les variables (comme dans [KMW1]). D'autre part, on se limite ici au cas où les potentiels sont réguliers alors que des singularités coulombiennes sont permises dans [KMW2] et que les potentiels sont coulombiens dans $[\mathrm{CT}]$ et $[\mathrm{Ra}]$.

En ce qui concerne l'approximation de Born-Oppenheimer, introduite dans [BO], on renvoie le lecteur aux références indiquées dans [KMSW] et [Jec1]. Pour l'approximation de Born-Oppenheimer dépendante du temps, de nombreux travaux ont été réalisés. On peut consulter à ce sujet $[\mathrm{H}]$ ainsi que les références citées dans ce mémoire.

Les détails de la méthode du commutateur de Mourre figurent dans [Mo] et [JMP]. Dans [T] et [W2], on trouvera des informations sur l'opérateur de Dirac. Enfin, signalons un travail récent (cf. [No]) sur l'amplitude de diffusion pour l'opérateur de Dirac avec champ magnétique, où des estimations semi-classiques de la résolvante sont établies.

Détaillons maintenant le système étudié. On considère une molécule diatomique à $N$ électrons. La mécanique quantique prédit que le comportement de cette molécule est donné par son opérateur d'énergie, l'opérateur auto-adjoint agissant dans $L^{2}\left(\mathbb{R}^{3(N+2)}\right)$,

$$
\tilde{H}=-\frac{1}{2 m_{1}} \Delta_{x_{1}}-\frac{1}{2 m_{2}} \Delta_{x_{2}}+\sum_{j=3}^{N+2}\left(-\frac{1}{2} \Delta_{x_{j}}\right)+\sum_{l<j} V_{i j}\left(x_{l}-x_{j}\right),
$$

où l'on a fixé la masse des électrons $(3 \leq j \leq N+2)$ à 1 ainsi que la constante de Planck. Les masses respectives des deux noyaux, $m_{1}$ et $m_{2}$, sont donc grandes devant 1, les fonctions réelles $V_{l j}$ représentent les interactions bilatérales entre particules. Plus généralement, on suppose que l'espace des configurations, dans lequel se meuvent les particules, est de dimension $n \geq 2$. L'opérateur précédent agit donc dans $L^{2}\left(\mathbb{R}^{n(N+2)}\right)$.

Soit $a=\left(A_{1}, A_{2}\right)$ une décomposition de $\{1, \ldots, N+2\}$ en deux amas telle que $j \in A_{j}$, pour $j \in\{1,2\}$. En effectuant un changement de variables convenable et en retirant le mouvement du centre de masse, on se ramène à l'étude de l'opérateur

$$
P(h)=-h^{2} \Delta_{x}+P^{a}(h)+I_{a}(h),
$$

agissant dans $L^{2}\left(\mathbb{R}^{n(N+1)}\right)$ (voir la partie 2 pour les expressions précises de $P^{a}(h)$ et $I_{a}(h)$ ). Le réel positif $h$ sera un petit paramètre (cf. (1)), l'hamiltonien interne $P^{a}(h)$ est la somme des opérateurs d'énergie de chaque amas, considérés comme isolés, et le potentiel interamas $I_{a}(h)$ rassemble les interactions entre particules appartenant à deux amas différents. La variable $x \in \mathbb{R}^{n}$ représente la position relative des centres de masse des amas. Ainsi 
l'opérateur $-h^{2} \Delta_{x}$ correspond à l'énergie cinétique du mouvement relatif de ces centres de masse.

Puisque le mouvement des particules légères, les électrons, doit être nettement plus rapide que celui des particules lourdes, les noyaux, il est naturel d'introduire un hamiltonien électronique correspondant à l'opérateur d'énergie du système constitué des $N$ électrons, en interaction entre eux et placés dans le champ extérieur créé par les noyaux, dont la position relative est liée au paramètre $x$. On considère donc la famille d'opérateurs $\left\{P_{e}(x ; h), x \in \mathbb{R}^{n}, h \leq h_{0}\right\}$ définis par

$$
P_{e}(x ; h)=P^{a}(h)+I_{a}(x ; h), \forall x \in \mathbb{R}^{n}, \forall h \leq h_{0} .
$$

On a

$$
P(h)=-h^{2} \Delta_{x}+P_{e}(h) .
$$

Les interactions bilatérales apparaissant dans ces opérateurs seront des fonctions $V \in$ $C^{\infty}\left(\mathbb{R}^{n} ; \mathbb{R}\right)$, vérifiant, pour un certain $\rho>0$,

$$
\forall \alpha \in \mathbb{N}^{n}, \exists C_{\alpha}>0 ; \forall x \in \mathbb{R}^{n},\left|\partial_{x}^{\alpha} V(x)\right| \leq C_{\alpha}\langle x\rangle^{-\rho-|\alpha|}
$$

(avec $\left.\langle x\rangle=\left(1+|x|^{2}\right)^{1 / 2}\right)$. On s'intéresse aux états du système dont l'évolution ressemble, asymptotiquement, à l'évolution libre d'états liés dans $A_{1}$ et $A_{2}$. Une telle évolution est donnée par la restriction à un sous-espace propre de $P^{a}(h)$ du propagateur de l'opérateur

$$
P_{a}(h) \equiv-h^{2} \Delta_{x}+P^{a}(h)
$$

Soient $E_{1}<\ldots<E_{r}$ les $r$ premières valeurs propres du spectre discret de $P^{a}(0)$, chaque $E_{j}$ étant de multiplicité $m_{j}$, pour $j \in\{1, \cdots, r\}$. Pour chaque $j$, on suppose qu'il y a exactement $m_{j}$ "courbes" $x \mapsto \lambda_{j l}(x ; 0)$, pour $l \in\left\{1, \cdots, m_{j}\right\}$, de valeurs propres $\lambda_{j l}(x ; 0)$ de $P_{e}(x ; 0)$ (répétées autant que leur multiplicité), qui tendent vers $E_{j}$ lorsque $|x| \rightarrow \infty$. De plus, on suppose que ces applications $x \mapsto \lambda_{j l}(x ; 0)$, à valeurs dans le spectre $\sigma\left(P_{e}(x ; 0)\right)$ de $P_{e}(x ; 0)$, sont globalement définies sur $\mathbb{R}^{n}$. Soit $\Pi_{j 0}(0)$ le projecteur spectral de $P^{a}(0)$, associé à $E_{j}$, et, pour tout $x \in \mathbb{R}^{n}$, soit $\Pi_{j}(x ; 0)$ celui de $P_{e}(x ; 0)$, associé aux $\lambda_{j l}(x ; 0)$, pour $l \in\left\{1, \cdots, m_{j}\right\}$.

Définition 1. Pour $\delta>0$ et pour tout $j \in\{1, \cdots, r\}$, on considère la condition $\left(H_{j, \delta}\right)$ suivante : il existe des fonctions $e_{j, \pm}$ et $E_{j, \pm}$ et des nombres réels $h_{j, \delta}>0$ tels que, pour tout $h \leq h_{j, \delta}$,

$$
\left\{\begin{array}{c}
\inf _{x \in \mathbb{R}^{n}}\left|E_{j, \pm}(x)-e_{j, \pm}(x)\right| \geq \delta . \\
\forall x \in \mathbb{R}^{n}, \quad e_{j,-}(x)<E_{j,-}(x)<e_{j,+}(x)<E_{j,+}(x), \\
\left.\forall x \in \mathbb{R}^{n}, \quad \lambda_{j 1}(x ; 0), \ldots, \lambda_{j m_{j}}(x ; 0) \in\right] E_{j,-}(x) ; e_{j,+}(x)[, \\
\forall x \in \mathbb{R}^{n}, \quad \sigma\left(P_{e}(x ; h)\right) \cap\left(\left[e_{j,-}(x) ; E_{j,-}(x)\right] \cup\left[e_{j,+}(x) ; E_{j,+}(x)\right]\right)=\emptyset .
\end{array}\right\}
$$


Sous la condition précédente, pour le même $\delta$ et pour $h_{\delta}=\min _{1 \leq j \leq r} h_{j, \delta}$, en notant par $\mathbb{1}_{] E_{j,-}(x), e_{j,+}(x)[}$ la fonction caractéristique de l'intervalle $] E_{j,-}(x), e_{j,+}(x)[$, on introduit la condition suivante. Il existe un $R_{0}>0$ tel que, pour tout $|x| \geq R_{0}$ et tout $h \in\left[0, h_{\delta}\right]$,

$$
\operatorname{dim} \operatorname{Im}\left(\mathbb{1}_{E_{j,-}(x), e_{j,+}(x)[}\left(P_{e}(x ; h)\right)\right)=m_{j} .
$$

Si ces conditions $\left(H_{j, \delta}\right)$ et $\left(H_{j, \delta}\right)^{\prime}$ sont satisfaites, pour un certain $\delta>0$, on dira que $E_{j}$ vérifie l'hypothèse de stabilité semi-classique.

Sous cette hypothèse de stabilité semi-classique, pour tout $j$, on peut trouver une famille $(\Gamma(x))_{x \in \mathbb{R}^{n}}$, de contours complexes indépendant de $h$ et entourant toutes les valeurs propres $\lambda_{j l}(x ; 0)\left(1 \leq l \leq m_{j}, 1 \leq j \leq r\right)$. Grâce à ces contours, on peut exprimer, au moyen d'une formule de Cauchy, le projecteur spectral $\Pi_{0}(0)$ de $P^{a}(0)$, associé aux valeurs propres $\left\{E_{1}, \ldots, E_{r}\right\}$, et le projecteur spectral $\Pi(x ; 0)$ de $P_{e}(x ; 0)$, associé aux valeurs propres $\left\{\lambda_{j l}(x ; 0), 1 \leq j \leq r, 1 \leq l \leq m_{j}\right\}$. Pour $h$ suffisamment petit, on peut définir un projecteur spectral $\Pi_{0}(h)$ (respectivement $\Pi(x ; h)$ ) de $P^{a}(h)$ (respectivement $\left.P_{e}(x ; h)\right)$ par la même formule de Cauchy (cf. partie 2$)$. Au moyen d'une intégrale directe, on définit un opérateur fibré $\Pi(h)$ par

$$
\Pi(h)=\int_{\mathbb{R}^{n}}^{\oplus} \Pi(x ; h) d x .
$$

Maintenant, on peut introduire la partie adiabatique de $P(h)$

$$
P^{A D}(h)=\Pi(h) P(h) \Pi(h) .
$$

Sous l'hypothèse $\left(D_{\rho}\right)$, pour $\rho>0$, l'opérateur $P^{A D}(h)$ est auto-ajoint (cf. [CDS]). On note par $R^{A D}(z ; h)$ sa résolvante et par $R(z ; h)$ celle de $P(h)$. L'objectif essentiel de ce travail est d'approcher la valeur au bord de la résolvante $R(z ; h)$ par celle de $R^{A D}(z ; h)$.

Avant d'énoncer le résultat principal de ce travail, on introduit des notions importantes.

Définition 2. Pour $j \in\{1, \cdots, r\}$, on dit que $E_{j}$ vérifie la condition de "noncroisement" si les valeurs propres, qui tendent vers $E_{j}$ lorsque $|x| \rightarrow \infty$, vérifient

$$
\forall x \in \mathbb{R}^{n}, \lambda_{j 1}(x ; 0)<\ldots<\lambda_{j l(j)}(x ; 0)
$$

pour un certain $l(j) \in\left\{1, \cdots, m_{j}\right\}$.

Définition 3. Étant donnés un hamiltonien classique $p: \mathbb{R}^{2 n} \longrightarrow \mathbb{R}$ et une énergie $E \in \mathbb{R}$, on note $\operatorname{par}^{-1}(E)$ la surface d'énergie $E$

$$
p^{-1}(E) \equiv\left\{(x, \xi) \in \mathbb{R}^{2 n} ; p(x, \xi)=E\right\} .
$$

On dit que l'énergie E est non-captive pour l'hamiltonien classique p si l'on a

$$
\forall(x, \xi) \in p^{-1}(E), \quad \lim _{t \rightarrow+\infty}\left\|\Phi^{t}(x, \xi)\right\|=\infty \quad \text { et } \quad \lim _{t \rightarrow-\infty}\left\|\Phi^{t}(x, \xi)\right\|=\infty
$$


$\Phi^{t}$ désignant le flot hamiltonien associé à $p$ et $\|\cdot\|$ la norme euclidienne sur $\mathbb{R}^{2 n}$. Un intervalle I est dit non-captif pour l'hamiltonien classique p si toute énergie $E \in I$ l'est.

Une fonction $a: \mathbb{R}^{2 n} \longrightarrow \mathbb{R}$, de classe $C^{1}$, est une fonction fuite globale pour l'hamiltonien classique $p$ à l'énergie $E \in \mathbb{R}$, s'il existe $\epsilon>0$ et $C>0$ tels que

$$
\forall(x, \xi) \in p^{-1}(] E-\epsilon, E+\epsilon[), \forall t \in \mathbb{R}, \frac{d}{d t}\left(\left(a \circ \Phi^{t}\right)(x, \xi)\right) \geq C .
$$

Notons que, dans ce cas, l'énergie E doit être non-captive pour $p$.

Théorème 4. On suppose que les potentiels vérifient $\left(D_{\rho}\right)$ pour $\rho>0$. Soit $E_{1}<\ldots<$ $E_{r} \in \sigma_{\text {disc }}\left(P^{a}(0)\right)$ les $r$ premières valeurs propres du spectre discret de $P^{a}(0)$. On suppose que l'hypothèse de stabilité semi-classique (cf. Définition 1) et la condition de "noncroisement" (cf. Définition 2) sont satisfaites pour tout $j$. Soit $E \notin\{0\} \cup\left\{E_{j}, 1 \leq j \leq r\right\}$ une énergie non-captive pour chaque hamiltonien classique $|\xi|^{2}+\lambda_{j l}(x ; 0)$, pour $1 \leq j \leq r$ et $1 \leq l \leq l(j)$ (cf. Définition 3).

1. Pour tout voisinage compact $\Lambda$ de E, assez petit, et pour tout $s>1 / 2$,

$$
\left\|\langle x\rangle^{-s} R^{A D}(\lambda \pm i 0 ; h)\langle x\rangle^{-s}\right\|=O\left(h^{-1}\right),
$$

pour $h$ assez petit, uniformément pour $\lambda \in \Lambda$.

2. On suppose que

$$
E<E^{A D} \equiv \inf _{x \in \mathbb{R}^{n}} \inf \left\{\sigma\left(P_{e}(x ; 0)\right) \backslash\left\{\lambda_{j l}(x ; 0), \forall j, l\right\}\right\} .
$$

Pour tout voisinage compact $\Lambda$ de $E$, assez petit, et pour tout $s>1 / 2$, on a, pour $h$ assez petit et uniformément pour $\lambda \in \Lambda$,

$$
\left\|\langle x\rangle^{-s}\left(R(\lambda \pm i 0 ; h)-\Pi R^{A D}(\lambda \pm i 0 ; h)\right)\langle x\rangle^{-s}\right\|=O(1)
$$

pour $\rho>1$, et

$$
\left\|\langle x\rangle^{-s} R(\lambda \pm i 0 ; h)\langle x\rangle^{-s}\right\|=O\left(h^{-1}\right)
$$

pour $\rho>0$.

Remarque 5. Dans [KMW1], il est établi que l'estimation (1) implique l'approximation (3) (et donc aussi l'estimation (4)) pour des potentiels à courte portée $(\rho>1)$. De plus, dans le Théorème 3.2 de [KMW1], des conditions suffisantes relatives à un opérateur conjugué sont dégagées pour obtenir l'estimation (1) et cette dernière est démontrée dans le cas où $r=1$. Ici, on obtient ces conditions suffisantes dans le cas où les valeurs propres $E_{j}$, pour $j>1$, ne sont pas simples (a priori).

D'autre part, signalons que la condition de non-capture sur E impose que, pour tout $j$,

$$
E \notin\left[\inf _{x, l} \lambda_{j l}(x ; 0) ; E_{j}\right]
$$


Pour l'étude semi-classique des opérateurs d'onde de canal (cf. Théorème 17) et pour celle des sections efficaces totales (cf. [Jec2]), lorsque $\rho>1$, il convient de pouvoir considérer une énergie $E$ telle que $E>E_{r}$. Dans ce cas, on a besoin de la condition

$$
E_{r}<E^{A D} \equiv \inf _{x \in \mathbb{R}^{n}} \inf \left\{\sigma\left(P_{e}(x ; 0)\right) \backslash\left\{\lambda_{j l}(x ; 0), \forall j, l\right\}\right\},
$$

pour avoir l'approximation (3) et donc l'estimation (4).

Remarque 6. En comparant ce résultat avec les estimations semi-classiques de [W1], on est en droit de se demander si cette condition de "non-croisement" est superflue.

Si un croisement a lieu dans la zone classiquement interdite, c'est-à-dire à une énergie strictement supérieure à $E$, i.e.

$$
\exists k \neq l ; C \equiv\left\{x \in \mathbb{R}^{n} ; \lambda_{j k}(x ; 0)=\lambda_{j l}(x ; 0)\right\} \neq \emptyset
$$

et il existe $\epsilon>0$ tel que, pour tout $x \in C$,

$$
\lambda_{j k}(x ; 0) \geq E+\epsilon
$$

il ne change pas le résultat (même si l'on doit introduire quelques modifications dans la preuve du Théorème 4, cf. Remarque 16). En revanche, si des énergies du croisement sont inférieures à E, il semble que l'on ne puisse pas toujours construire une fonction "multi-fuite" globale.

Le problème pourrait être éclairci par l'étude des résonances pour des opérateurs de Schrödinger à potentiel matriciel avec croisement des valeurs propres $\lambda_{j}(x)$ du potentiel, comme dans [Ne], mais près d'une énergie non-captive pour chaque hamiltonien classique $|\xi|^{2}+\lambda_{j}(x)$. Pour un autre aspect de ce problème, voir la Remarque 1.3 dans [Jec2].

A partir de l'approximation (3) du Théorème 4 (donc pour $\rho>1$ ), on établit, dans la bande d'énergie $\left[E_{r}, E^{A D}\right]$, l'approximation des opérateurs d'onde de canal

$$
\Omega_{ \pm}(h)=s-\lim _{t \rightarrow \pm \infty} e^{i h^{-1} t P(h)} e^{-i h^{-1} t P_{a}(h)} \Pi_{0}(h)
$$

(qui existent, cf. [RS3]) par les opérateurs d'onde adiabatiques

$$
\Omega_{ \pm}^{A D}(h)=s-\lim _{t \rightarrow \pm \infty} e^{i h^{-1} t P^{A D}(h)} e^{-i h^{-1} t P_{a}(h)} \Pi_{0}(h) .
$$

Ce résultat (cf. Théorème 17) constitue une amélioration du résultat correspondant dans [KMW1]. Signalons qu'une autre amélioration a été obtenue dans [KMW2], où des singularités coulombiennes dans les potentiels sont permises.

Pour la preuve du Théorème 4, on adopte la stratégie suivie dans [KMW1]. Cette stratégie a été utilisée pour la première fois dans $[\mathrm{GM}]$ pour obtenir une preuve rapide de l'estimation semi-classique de la résolvante d'opérateurs de Schrödinger à deux corps, initialement établie dans $[\mathrm{RT}]$. Pour obtenir une estimation semi-classique de la résolvante 
d'un opérateur de Schrödinger $H$, on utilise la méthode du commutateur de Mourre (cf. [Mo], [JMP]). Il s'agit donc de trouver un opérateur conjugué $A$ de sorte que le commutateur $i h^{-1}[H, A]$ soit strictement positif, dans un certain sens. L'idée développée dans [GM] consiste à construire une fonction fuite globale (cf. Définition 3) pour l'hamiltonien classique associé à $H$ et, en gros, de choisir comme opérateur conjugué l'opérateur pseudo-différentiel de Weyl de symbole la fonction fuite globale.

Dans le présent travail, on introduit un type particulier de fonction fuite globale. Étant donnée une famille d'hamiltoniens classiques, on veut construire une fonction, qui soit une fonction fuite globale pour chaque hamiltonien classique, une fonction "multi-fuite" globale. Pour les hamiltoniens classiques $|\xi|^{2}+\lambda_{j l}(x ; 0)(1 \leq j \leq r, 1 \leq l \leq l(j))$, intervenant dans le Théorème 4 , on utilise la condition de "non-croisement", introduite dans la Définition 2, pour prouver l'existence d'une telle fonction. Pourquoi ne pas simplement utiliser une fonction fuite pour chaque hamiltonien classique? Parce que, dans ce cas, un terme particulier, figurant dans l'estimation de Mourre semi-classique, semble incontrôlable (cf. Remarque 14). A ce sujet, voir aussi la Remarque 21.

Une telle fonction "multi-fuite" globale semble être un bon outil pour obtenir des estimations semi-classiques de résolvante pour des opérateurs matriciels. Par exemple, on établit une telle estimation pour la résolvante d'un opérateur de Schrödinger à deux corps avec un potentiel matriciel à longue portée. Pour l'opérateur de Dirac avec champ électrique scalaire à longue portée, on obtient également un contrôle semi-classique de la résolvante, comme dans [Ce], mais sous des hypothèses plus faibles sur le potentiel. C'est l'objet du

Théorème 7. On utilise les notations de la partie 5. En particulier, on note par D l'opérateur de Dirac. On suppose que le champ électrique scalaire $V$ est réel et satisfait la condition $\left(D_{\rho}\right)$ pour un certain $\rho>0$. Soit $\lambda_{0}>1$ une énergie non-captive (cf. Définition 3) pour l'hamiltonien classique $\langle\xi\rangle+V(x)$ telle que

$$
\lambda_{0}>\left(\inf _{x \in \mathbb{R}^{3}} V(x)\right)-1 .
$$

Pour tout voisinage compact $\Lambda$ de $\lambda_{0}$, assez petit, et pour tout $s>1 / 2$,

$$
\left\|\langle x\rangle^{-s}\left((\lambda \pm i 0) I_{4}-D\right)^{-1}\langle x\rangle^{-s}\right\|=O\left(h^{-1}\right)
$$

pour $h$ assez petit et uniformément pour $\lambda \in \Lambda$.

Remarque 8. Ce Théorème 7 est encore valable si $\lambda_{0}<-1$ est non-captive pour l'hamiltonien classique $-\langle\xi\rangle+V(x)$ et vérifie

$$
\lambda_{0}<\left(\inf _{x \in \mathbb{R}^{3}} V(x)\right)+1
$$

Ce travail est organisé de la façon suivante. Dans la partie 2, on rappelle des propriétés de base, obtenues dans [KMW1], sur les projecteurs $\Pi(x ; h)$. La partie 3 est dévolue à la preuve du Théorème 4. On obtient l'approximation adiabatique des opérateurs d'onde 
de canal dans la partie 4. Enfin, dans la partie 5, on considère le cas d'un opérateur de Schrödinger matriciel et on améliore le résultat de [Ce] sur l'opérateur de Dirac.

\section{REMERCIEMENTS.}

L'auteur exprime sa profonde gratitude envers X.P. Wang, pour son soutient constant et ses nombreux conseils (en particulier, il signala que la méthode utilisée dans la partie 3 devait s'appliquer à l'opérateur de Dirac). L'auteur remercie J.M. Combes et A. Martinez pour leurs remarques et l'intérêt qu'ils ont porté à ce travail.

L'auteur tient aussi à remercier les membres du département de mathématiques de la TU Berlin pour leur hospitalité et en particulier V. Bach.

L'auteur est soutenu financièrement par le programme européen TMR de la Commission Européenne, intitulé :" Network Postdoctoral training programme in partial differential equations and application in quantum mechanics".

\section{Table des matières}

\section{Introduction.}

2 Préliminaires.

3 Estimation semi-classique de la résolvante de $P$.

4 Approximation semi-classiques des opérateurs d'onde de canal.

5 Autres utilisations d'une fonction fuite ou "multi-fuite" globale.

\section{Préliminaires.}

Dans cette partie, on introduit des notations et on établit quelques propriétés de l'hamiltonien électronique. En particulier, ces propriétés permettent de définir $P^{A D}$ comme opérateur auto-adjoint (cf. [CDS]). Les arguments utilisés ici proviennent essentiellement de [KMW1].

Tout d'abord, donnons l'expression exacte des opérateurs $P^{a}(h)$ et $I_{a}(x ; h)$. Tandis que la variable $x \in \mathbb{R}^{n}$ repère la position relative des centres de masse des amas, on prend des coordonnées atomiques dans chaque amas. On obtient ainsi $N$ variables internes que l'on désigne par $y \in \mathbb{R}^{n N}$. Pour $k \in\{1,2\}$, notons par $A_{k}^{\prime}$ l'ensemble des électrons de l'amas $A_{k}$, par $\left|A_{k}^{\prime}\right|$ le cardinal de cet ensemble et par $M_{k}=m_{k}+\left|A_{k}^{\prime}\right|$ la masse totale de l'amas 
$A_{k}$. Le petit paramètre $h$ est alors donné par

$$
h=\left(\frac{1}{2 M_{1}}+\frac{1}{2 M_{2}}\right)^{1 / 2} .
$$

On a

$$
P^{a}(h)=\sum_{k=1}^{2}\left[\sum_{j \in A_{k}^{\prime}}\left(-\frac{1}{2} \Delta_{y_{j}}+V_{k j}\left(y_{j}\right)\right)-\frac{1}{2 m_{k}} \sum_{l, j \in A_{k}^{\prime}} \nabla_{y_{l}} \cdot \nabla_{y_{j}}+\frac{1}{2} \sum_{l, j \in A_{k}^{\prime}} V_{l j}\left(y_{l}-y_{j}\right)\right]
$$

et

$$
\begin{gathered}
I_{a}(x ; h)=\sum_{l \in A_{1}^{\prime}, j \in A_{2}^{\prime}} V_{l j}\left(y_{l}-y_{j}+x+f_{2}-f_{1}\right)+\sum_{l \in A_{1}^{\prime}} V_{l 2}\left(x-f_{1}+f_{2}-y_{l}\right) \\
\quad+\sum_{j \in A_{2}^{\prime}} V_{1 j}\left(x-f_{1}+f_{2}-y_{j}\right)+V_{12}\left(x-f_{1}+f_{2}\right),
\end{gathered}
$$

où les quantités $f_{k}=\frac{1}{M_{k}} \sum_{j \in A_{k}^{\prime}} y_{j}$, pour $k \in\{1,2\}$, dépendent de $h$. Pour $l<j$, on a posé $V_{j l}(z)=V_{l j}(-z)$. Notons par $P_{H E}$ le terme de Hughes-Eckart suivant

$$
P_{H E} \equiv-\sum_{k=1}^{2} \frac{1}{2 m_{k}} \sum_{l, j \in A_{k}^{\prime}} \nabla_{y_{l}} \cdot \nabla_{y_{j}}
$$

(le "." désigne le produit scalaire des gradients).

Comme l'opérateur $P^{a}(h)$ converge vers $P^{a}(0)$, lorsque $h \rightarrow 0$, en norme des résolvantes, il existe, pour tout $j, m_{j}$ valeurs propres $E_{j l}(h)$ de $P^{a}(h)$ qui convergent vers $E_{j}$, quand $h \rightarrow 0$. Soit $\Pi_{j 0}(h)$ le projecteur spectral de $P^{a}(h)$ associé à ces $m_{j}$ valeurs propres. Rappelons que, dans l'introduction, on a supposé que, pour tout $j, m_{j}$ valeurs propres $\lambda_{j l}(x ; 0)$ de $P_{e}(x ; 0)$ tendent vers $E_{j}$.

Sous l'hypothèse de stabilité semi-classique (cf. Définition 1), pour tout $j$, on peut trouver $\left(\Gamma_{j}(x)\right)_{x \in \mathbb{R}^{n}}$, une famille de contours complexes, indépendants de $h$, qui entourent tous les $\lambda_{j l}(x ; 0)\left(1 \leq l \leq m_{j}\right)$ de sorte que l'on ait

$$
\inf _{x \in \mathbb{R}^{n}} d\left(\sigma\left(P_{e}(x ; h)\right), \Gamma_{j}(x)\right) \geq \delta / 2
$$

pour tout $h \in\left[0 ; h_{\delta}\right]$. Comme $\lambda_{j l}(x ; 0) \rightarrow E_{j}(|x| \rightarrow \infty)$, on est en droit de choisir ces contours tels que

$$
\Gamma_{j}(x)=\left\{z \in \mathbb{C} ;\left|z-E_{j}\right|=\delta / 2\right\} \equiv \Gamma_{j}(\infty)
$$

pour $|x| \geq R_{1}$, pour un certain $R_{1} \geq R_{0}$, indépendant de $j$. Ainsi, pour $\delta$ et $h_{\delta}$ assez petits, on peut écrire, pour tout $h \in\left[0 ; h_{\delta}\right]$,

$$
\Pi_{j 0}(h)=\frac{1}{2 i \pi} \int_{\Gamma_{j}(\infty)}\left(z-P^{a}(h)\right)^{-1} d z
$$


et, pour tout $x \in \mathbb{R}^{n}$, le projecteur spectral de $P_{e}(x ; 0)$, associé aux valeurs propres $\lambda_{j l}(x ; 0)\left(1 \leq l \leq m_{j}\right)$, est donné, grâce à $\left(H_{j, \delta}\right)$, par

$$
\Pi_{j}(x ; 0)=\frac{1}{2 i \pi} \int_{\Gamma_{j}(x)}\left(z-P_{e}(x ; 0)\right)^{-1} d z .
$$

En s'appuyant encore sur $\left(H_{j, \delta}\right)$, on définit, pour tout $x \in \mathbb{R}^{n}$ et tout $h \in\left[0 ; h_{\delta}\right]$,

$$
\Pi_{j}(x ; h)=\frac{1}{2 i \pi} \int_{\Gamma_{j}(x)}\left(z-P_{e}(x ; h)\right)^{-1} d z .
$$

D'après $\left(H_{j, \delta}\right)^{\prime}$, l'opérateur $\Pi_{j}(x ; h)$ est, pour $|x|$ assez grand, le projecteur spectral de $P_{e}(x ; h)$, associé à certaines valeurs propres $\lambda_{j 1}(x ; h), \ldots, \lambda_{j m_{j}}(x ; h)$, dont la multiplicité totale est $m_{j}$. Dans la Proposition 11, on montre que ces valeurs propres sont globalement définies et proches des $\lambda_{j 1}(x ; 0), \ldots, \lambda_{j m_{j}}(x ; 0)$.

De façon analogue, on peut trouver $(\Gamma(x))_{x \in \mathbb{R}^{n}}$, une famille de contours complexes, indépendants de $h$, qui entourent tous les $\lambda_{j l}(x ; 0)\left(1 \leq l \leq m_{j}, 1 \leq j \leq r\right)$. On peut également les choisir de sorte que l'on ait, pour $|x|$ assez grand,

$$
\Gamma(x)=\left\{z \in \mathbb{C} ; \inf _{1 \leq j \leq r}\left|z-E_{j}\right|=\delta / 2\right\} \equiv \Gamma(\infty) .
$$

Comme dans l'introduction, on pose, pour $h$ assez petit,

$$
\begin{aligned}
\Pi_{0}(h) & =\frac{1}{2 i \pi} \int_{\Gamma(\infty)}\left(z-P^{a}(h)\right)^{-1} d z, \\
\Pi(x ; h) & =\frac{1}{2 i \pi} \int_{\Gamma(x)}\left(z-P_{e}(x ; h)\right)^{-1} d z .
\end{aligned}
$$

En particulier, on a, pour tout $(x ; h)$,

$$
\Pi_{0}(h)=\sum_{j=1}^{r} \Pi_{j 0}(h) \text { et } \Pi(x ; h)=\sum_{j=1}^{r} \Pi_{j}(x ; h) .
$$

Donnons maintenant des propriétés de régularité et de décroissance à l'infini de ces projecteurs. A partir des formules de Cauchy précédentes, on voit que ce sont des fonctions $C^{\infty}$ car les potentiels le sont. Grâce à la décroissance exponentielle des fonctions propres de l'opérateur $P^{a}(0)$ associées aux valeurs propres $E_{j}$ (cf. [A]), ces projecteurs possèdent les propriétés suivantes.

Proposition 9. Sous la condition $\left(D_{\rho}\right)(\rho>0)$ et sous l'hypothèse de stabilité semiclassique (cf. Définition 1), on a les estimations suivantes, pour tout $1 \leq j \leq r$,

$$
\begin{gathered}
\forall \alpha \in \mathbb{N}^{n}, \exists D_{\alpha}>0 ; \forall x \in \mathbb{R}^{n},\left\|\partial_{x}^{\alpha}\left(\Pi_{j}(x ; h)-\Pi_{j 0}(h)\right)\right\| \leq D_{\alpha}\langle x\rangle^{-\rho-|\alpha|}, \\
\forall \alpha \in \mathbb{N}^{n}, \exists D_{\alpha}>0 ; \forall x \in \mathbb{R}^{n},\left\|\left(\partial_{x}^{\alpha} I_{a}\right)(x ; h) \Pi_{j 0}(h)\right\|+\left\|\left(\partial_{x}^{\alpha} I_{a}\right)(x ; h) \Pi_{j}(x ; h)\right\| \leq D_{\alpha}\langle x\rangle^{-\rho-|\alpha|},
\end{gathered}
$$




$$
\left\|\Pi_{j}(x ; h) P_{e}(x ; h) \Pi_{j}(x ; h)-\Pi_{j}(x ; h) P^{a}(h) \Pi_{j 0}(h)\right\|=O\left(\langle x\rangle^{-\rho}\right),
$$

uniformément pour $h \in\left[0, h_{\delta}\right], h_{\delta}$ assez petit. Ici, $\|\cdot\|$ désigne la norme de $\mathcal{L}\left(L^{2}\left(\mathbb{R}_{y}^{n N}\right)\right)$. Les opérateurs $\Pi(x ; h)$ vérifient des propriétés analogues.

Démonstration : On suit essentiellement les arguments de [KMW1]. Voir [Jec1].

Remarque 10. Signalons une propriété importante, commune aux fonctions régulières à valeurs projecteur. Pour tout $(x ; h)$,

$$
\Pi(x ; h)\left(\nabla_{x} \Pi\right)(x ; h) \Pi(x ; h)=0 .
$$

En effet, puisqu'il s'agit de projecteurs, on peut écrire

$$
0=\Pi(x ; h)\left[\nabla_{x}\left(\Pi^{2}(x ; h)-\Pi(x ; h)\right)\right] \Pi(x ; h)=\Pi(x ; h)\left(\nabla_{x} \Pi\right)(x ; h) \Pi(x ; h) .
$$

Comme on l'a déjà signalé, on va voir que les valeurs propres $\lambda_{j 1}(x ; h), \ldots, \lambda_{j m_{j}}(x ; h)$ se prolongent à $\mathbb{R}^{n}$ et que l'on sait les localiser. C'est l'objet de la

Proposition 11. Pour tout $1 \leq j \leq r$, on suppose que $E_{j}$ vérifie l'hypothèse de stabilité semi-classique (cf. Définition 1) pour un réel $\delta>0$. Pour $h \in\left[0, h_{\delta}\right], h_{\delta}$ assez petit, pour tout $x \in \mathbb{R}^{n}$, il existe alors exactement $m_{j}$ valeurs propres de $P_{e}(x ; h)$, $\lambda_{j 1}(x ; h), \ldots, \lambda_{j m_{j}}(x ; h)$, chacune répétée autant que sa multiplicité, telles que

$$
\forall l \in<1, m>, \lambda_{j l}(x ; h)=\lambda_{j l}(x ; 0)+O\left(h^{2}\right),
$$

uniformément en $x$. Le spectre de l'opérateur $P_{e}(x ; h)$ possède donc la propriété suivante. Pour tout $1 \leq j \leq r$, pour tout $x \in \mathbb{R}^{n}$ et tout $h \in\left[0, h_{\delta}\right]$,

$$
d\left(\left\{\lambda_{j 1}(x ; h), \ldots, \lambda_{j m_{j}}(x ; h)\right\}, \sigma\left(P_{e}(x ; h)\right) \backslash\left\{\lambda_{j 1}(x ; h), \ldots, \lambda_{j m_{j}}(x ; h)\right\}\right) \geq \delta / 3 .
$$

Démonstration : On reprend la preuve de [KMW1] et on renvoie pour certaines étapes à l'annexe $\mathrm{D}$ de [Jec1]. Cette proposition est basée sur l'estimation

$$
\left\|\Pi_{j}(x ; h)-\Pi_{j}(x ; 0)\right\|=O\left(h^{2}\right), \text { uniformément en } x .
$$

D'après la relation (4), on est ramené à étudier, pour $z \in \Gamma_{j}(x)$, l'opérateur

$$
\left(P_{e}(x ; h)-P_{e}(x ; 0)\right)\left(z-P_{e}(x ; h)\right)^{-1} .
$$

Notons tout de suite que, uniformément en $x$ et $z \in \Gamma(x)$,

$$
\left\|P_{H E}\left(z-P_{e}(x ; h)\right)^{-1}\right\|=O\left(h^{2}\right) .
$$


Il reste donc à examiner la contribution du potentiel inter-amas $I_{a}$ qui est du type

$$
\left[V\left(x+L_{h}(y)+L(y)\right)-V(x+L(y))\right]\left(z-P_{e}(x ; h)\right)^{-1}
$$

où $L_{h}, L$ sont des applications linéaires de $\mathbb{R}^{n N}$ dans $\mathbb{R}^{n}$ avec $\left\|L_{h}\right\|=O\left(h^{2}\right)$ et $L$ indépendant de $h$, et où $V$ vérifie $\left(D_{\rho}\right)$. On pense utiliser une formule de Taylor en $h$. On voit alors apparaître un terme $L_{h}(y)$ que l'on "absorbe" en projettant sur $\operatorname{Im} \Pi_{j}(x ; h)$, grâce à la décroissance exponentielle des fonctions propres de $P^{a}(0)$, associées à la valeur propre $E_{j}$ (cf. [A]). On obtient ainsi

$$
\left\|\left(\Pi_{j}(x ; h)-\Pi_{j}(x ; 0)\right) \Pi_{j}(x ; h)\right\|+\left\|\left(\Pi_{j}(x ; h)-\Pi_{j}(x ; 0)\right) \Pi_{j}(x ; 0)\right\|=O\left(h^{2}\right),
$$

d'où l'on tire (11), puisque l'on manipule des projecteurs.

Pour $h$ assez petit, $\Pi_{j}(x ; h)$ et $\Pi_{j}(x ; 0)$ ont même rang, pour tout $x \in \mathbb{R}^{n}$. Le projecteur $\Pi_{j}(x ; h)$ est donc le projecteur spectral de $P_{e}(x ; h)$ associé à $\lambda_{j 1}(x ; h), \ldots, \lambda_{j m_{j}}(x ; h), m_{j}$ valeurs propres, chacune répétée autant que sa multiplicité. L'hypothèse de stabilité semiclassique implique la propriété $(10)$ pour $P_{e}(x ; h)$.

On montre maintenant que les $\lambda_{j l}(x ; h)$ sont proches des $\lambda_{j l}(x ; 0)$. Des arguments précédents résulte aussi l'estimation

$$
\left\|\left(P_{e}(x ; h)-P_{e}(x ; 0)\right) \Pi_{j}(x ; 0)\right\|+\left\|\left(P_{e}(x ; h)-P_{e}(x ; 0)\right) \Pi_{j}(x ; h)\right\|=O\left(h^{2}\right),
$$

qui permet, conjointement avec (11), d'écrire

$$
\left\|\Pi_{j}(x ; h) P_{e}(x ; h) \Pi_{j}(x ; h)-\Pi_{j}(x ; 0) P_{e}(x ; 0) \Pi_{j}(x ; 0)\right\|=O\left(h^{2}\right) .
$$

En utilisant une formule de "minimax", on obtient le résultat cherché (cf. [Jec1]).

Sous l'hypothèse de "non-croisement" pour chaque $j$, il y a $l(j)$ fonctions $\lambda_{j l}(\cdot ; 0)$ qui tendent vers $E_{j}$ à l'infini et chaque $\lambda_{j l}(\cdot ; 0)$ est de multiplicité constante. On peut donc contruire une famille $\left\{\Gamma_{j l}(x), x \in \mathbb{R}^{n}\right\}$ de contours dans $\mathbb{C}$ telle que

$$
\Pi_{j l}(x ; 0)=\frac{1}{2 i \pi} \int_{\Gamma_{j l}(x)}\left(z-P_{e}(x ; 0)\right)^{-1} d z
$$

soit le projecteur spectral associé à $\lambda_{j l}(x ; 0)$. Ces projecteurs sont aussi de classe $C^{\infty}$ ainsi que les valeurs propres correspondantes en vertu de la relation

$$
\lambda_{j l}(x ; 0)=\frac{1}{m_{j}} \operatorname{Tr}\left(\Pi_{j l}(x ; 0) P_{e}(x ; 0)\right)
$$

où $\operatorname{Tr}$ désigne la trace. A la différence des contours précédents, le périmètre de ceux-ci tend vers 0 lorsque $x$ tend vers l'infini (si la valeur propre $E_{j}$ est multiple). Malgré cela, ces valeurs propres $\lambda_{j l}(x ; 0)$ vérifient la condition $\left(D_{\rho}^{\prime}\right)$ suivante

$$
\forall \alpha \in \mathbb{N}^{n} ;|\alpha| \leq 1, \exists C_{\alpha}>0 ; \forall x \in \mathbb{R}^{n},\left|\partial_{x}^{\alpha}\left(\lambda_{j l}(x ; 0)-E_{j}\right)\right| \leq C_{\alpha}\langle x\rangle^{-\rho-|\alpha|},\left(D_{\rho}^{\prime}\right)
$$


(il s'agit du $\rho$ de l'hypothèse $\left(D_{\rho}\right)$ vérifiée par les potentiels). Pour confirmer ce point, on va s'appuyer sur $[\mathrm{K}]$. Comme dans la preuve de la Proposition 11 , les $\lambda_{j}(x ; 0)$ sont, pour $|x|$ assez grand, les valeurs propres de la matrice symétrique $M(x)$ représentant $P_{e}(x ; 0)$ dans la base $\left(\Pi(x ; 0) \phi_{k}\right)_{1 \leq k \leq m}$, où $\left(\phi_{k}\right)_{1 \leq k \leq m}$ est une base orthonormée de $\operatorname{Im} \Pi_{0}(0)$. Les coefficients $a_{k l}(x)$ de $M(x)$ vérifient

$$
\left|\partial_{x}^{\alpha}\left(a_{k l}(x)-\delta_{k l}\right)\right|=O\left(\langle x\rangle^{-\rho-|\alpha|}\right)
$$

pour tout $\alpha \in \mathbb{N}^{n}$ grâce à la Proposition 9 . D'après $[\mathrm{K}]$ page 111 , les $\lambda_{j}(x ; 0)$ vérifient la condition $\left(D_{\rho}^{\prime}\right)$.

Enfin, l'opérateur $P(h)$ est auto-adjoint sur le domaine du laplacien dans $L^{2}\left(\mathbb{R}^{n(N+1)}\right)$, noté $D(P(h)$ ). En utilisant la Proposition 9 et les arguments de [CDS], on vérifie (cf. [Jec1]) que les opérateurs

$$
P^{A D}(h)=\Pi(h) P(h) \Pi(h) \text { et } P_{j}^{A D}(h)=\Pi_{j}(h) P(h) \Pi_{j}(h),
$$

pour tout $j$, sont auto-adjoints sur les domaines respectifs

$$
\begin{aligned}
& \left\{\phi \in L^{2}\left(\mathbb{R}^{n(N+1)}\right) ; \Pi(h) \phi \in D(P(h))\right\}, \\
& \left\{\phi \in L^{2}\left(\mathbb{R}^{n(N+1)}\right) ; \Pi(h) \phi \in D(P(h))\right\} .
\end{aligned}
$$

\section{Estimation semi-classique de la résolvante de $P$.}

L'objet de cette partie est d'établir, dans les conditions de la partie 2 précédente, l'approximation adiabatique de la résolvante totale pour certaines énergies (cf. Théorème 4). A la différence de [KMW1] où le projecteur $\Pi$ est de rang 1 , on est amené ici à construire une fonction "multi-fuite" globale, c'est-à-dire une fonction qui est simultanément une fonction fuite globale pour plusieurs hamiltoniens classiques.

Pour obtenir le Théorème 4, on montre l'estimation de Mourre suivante.

Proposition 12. On suppose que les potentiels vérifient $\left(D_{\rho}\right)$ pour un réel $\rho>0$. Soit $E_{1}<\ldots<E_{r} \in \sigma_{\text {disc }}\left(P^{a}(0)\right)$ vérifiant chacune l'hypothèse de stabilité semi-classique (cf. Définition 1) et l'hypothèse de "non-croisement" (cf. Définition 2). Pour toute énergie $E \notin\{0\} \cup\left\{E_{j}, 1 \leq j \leq r\right\}$, non-captive pour chaque hamiltonien classique $|\xi|^{2}+\lambda_{j l}(x ; 0)$ (cf. Définition 3), $1 \leq j \leq r$ et $1 \leq l \leq l(j)$, il existe un opérateur $F^{A D}(h)$ tel que

$$
\left\|\left[\left[P^{A D}(h), F^{A D}(h)\right], F^{A D}(h)\right]\left(P^{A D}(h)+i\right)^{-1}\right\|=O\left(h^{2}\right),
$$

qui vérifie l'estimation de Mourre

$$
\chi\left(P^{A D}(h)\right) i\left[P^{A D}(h), F^{A D}(h)\right] \chi\left(P^{A D}(h)\right) \geq \alpha h \chi^{2}\left(P^{A D}(h)\right)
$$

avec $\chi=\mathbb{1}_{E-\delta, E+\delta[}, \delta>0$ et $\alpha>0$ indépendants de $h$. 
Démonstration : L'hypothèse de non-capture impose, pour tout $j$,

$$
E \notin\left[\inf _{x, l} \lambda_{j l}(x ; 0) ; E_{j}\right]
$$

Soit $s$ le plus grand entier $j$ tel que $E_{j}<E$. On adopte la stratégie utilisée dans [KMW1]. Pour chaque hamiltonien classique $|\xi|^{2}+\lambda_{j l}(x ; 0)-E_{j}$, il est naturel de construire une fonction fuite globale (cf. Définition 3) comme dans [GM]. Cependant, on préfère construire ici une fonction $a$ qui soit une fonction fuite globale pour chaque hamiltonien classique $|\xi|^{2}+\lambda_{j l}(x ; 0)-E_{j}(j \leq s)$ à l'énergie $E-E_{j}>0$. Sous les hypothèses de cette Proposition 12, l'existence d'une telle fonction "multi-fuite" globale est donnée par le

Lemme 13. On considère des potentiels $\left(V_{j}\right)_{1 \leq j \leq q}$ de $C^{\infty}\left(\mathbb{R}^{n} ; \mathbb{R}\right)$, vérifiant $\left(D_{\rho}^{\prime}\right)$ pour un réel $\rho>0$. Pour tout $j$, soit $\lambda_{j}>0$ une énergie de non-capture pour l'hamiltonien classique $p_{j}(x, \xi)=|\xi|^{2}+V_{j}(x)$. Sous l'hypothèse de "non-croisement"

$$
j \neq k \Longrightarrow\left(\forall x \in \mathbb{R}^{n}, V_{j}(x)-\lambda_{j} \neq V_{k}(x)-\lambda_{k}\right)
$$

il existe une fonction qui est, simultanément pour tout $j$, une fonction fuite globale à l'énergie $\lambda_{j}$ pour l'hamiltonien $p_{j}$ (cf. Définition 3).

Démonstration : D'après [GM], il existe $R>0, C_{0}>0$ et $\epsilon>0$ tels que, pour tout $j$,

- $\left\{p_{j}, x \cdot \xi\right\} \geq \lambda_{j} / 2$ pour $|x| \geq R$ et $(x, \xi) \in p_{j}^{-1}(] \lambda_{j}-\epsilon ; \lambda_{j}+\epsilon[)$,

- il existe $a_{j} \in C^{\infty}\left(\mathbb{R}^{2 n}\right)$ de la forme :

$$
a_{j}(x, \xi)=x \cdot \xi+C_{j} \chi_{j}(x) f_{j}(x, \xi)
$$

où $C_{j}>0, \chi_{j} \in C_{0}^{\infty}\left(\mathbb{R}^{n}\right), \chi_{j} f_{j} \in C_{0}^{\infty}\left(\mathbb{R}^{2 n}\right)$, et telle que

$$
\left\{p_{j}, a_{j}\right\} \geq C_{0}
$$

$\operatorname{sur} p_{j}^{-1}(] \lambda_{j}-\epsilon ; \lambda_{j}+\epsilon[)$.

Soient $R^{\prime} \equiv \max _{j} \sup \left\{|x| ; x \in \operatorname{supp}_{j}\right\}>R$ et $R$ " $>R^{\prime}$. Grâce à l'hypothèse de "noncroisement" (3), on va montrer qu'il existe $\alpha>0$ tel que, pour tout $\epsilon$ assez petit et pour $\left(V_{j}, \lambda_{j}\right) \neq\left(V_{k}, \lambda_{k}\right)$, la distance

$d\left(\left\{(x, \xi) ;|x| \leq R^{\prime \prime}\right\} \cap p_{j}^{-1}(] \lambda_{j}-\epsilon ; \lambda_{j}+\epsilon[),\{(x, \xi) ;|x| \leq R "\} \cap p_{k}^{-1}(] \lambda_{k}-\epsilon ; \lambda_{k}+\epsilon[)\right) \geq \alpha$.

Posons

$$
K=\max _{j} \sup _{|x| \leq R^{\prime \prime}}\left|V_{j}(x)\right| \text { et } K_{j}=\sup _{|x| \leq R^{\prime \prime}}\left\|V_{j}^{\prime}(x)\right\| .
$$

D'après (3), il existe un $\delta>0$ tel que

$$
|x| \leq R=\left(\forall j \neq k,\left|V_{j}(x)-\lambda_{j}-V_{k}(x)+\lambda_{k}\right| \geq \delta\right)
$$


Prenons $\epsilon<\delta / 2$ et $\eta, r>0$. Supposons qu'il existe des couples

$$
\begin{aligned}
& (x, \xi) \in\left\{(y, \eta) ;|y| \leq R^{\prime \prime}\right\} \cap p_{j}^{-1}(] \lambda_{j}-\epsilon ; \lambda_{j}+\epsilon[), \\
& \left(x^{\prime}, \xi^{\prime}\right) \in\left\{(y, \eta) ;|y| \leq R^{\prime \prime}\right\} \cap p_{k}^{-1}(] \lambda_{k}-\epsilon ; \lambda_{k}+\epsilon[)
\end{aligned}
$$

pour $j \neq k$, tels que $\left|\xi-\xi^{\prime}\right|<\eta,\left|x-x^{\prime}\right|<r$. On a donc

$$
\left.|| \xi^{\prime}\right|^{2}-|\xi|^{2}|\leq| \xi^{\prime}-\xi|\cdot| \xi^{\prime}+\xi \mid \leq \eta\left(\left(\lambda_{j}+\epsilon+K\right)^{1 / 2}+\left(\lambda_{k}+\epsilon+K\right)^{1 / 2}\right) .
$$

D'une part,

$$
\begin{aligned}
\left|V_{j}(x)-\lambda_{j}-\left(V_{k}\left(x^{\prime}\right)-\lambda_{k}\right)\right| & \leq\left|p_{j}(x, \xi)-\lambda_{j}-\left(p_{k}\left(x^{\prime}, \xi^{\prime}\right)-\lambda_{k}\right)\right|+\left.|| \xi^{\prime}\right|^{2}-|\xi|^{2} \mid \\
& \leq 2 \epsilon+\eta\left(\left(\lambda_{j}+\epsilon+K\right)^{1 / 2}+\left(\lambda_{k}+\epsilon+K\right)^{1 / 2}\right)
\end{aligned}
$$

et d'autre part,

$\left|V_{j}(x)-\lambda_{j}-\left(V_{k}\left(x^{\prime}\right)-\lambda_{k}\right)\right| \geq\left|V_{j}\left(x^{\prime}\right)-\lambda_{j}-\left(V_{k}\left(x^{\prime}\right)-\lambda_{k}\right)\right|-\left|V_{j}(x)-V_{j}\left(x^{\prime}\right)\right| \geq \delta-r K_{j}$.

Pour $r$ et $\eta$ assez petits, on a une contradiction. Par conséquent, il existe un $\alpha>0$ pour lequel l'implication (4) est vraie, pour tout $\epsilon$ assez petit.

Grâce à cette propriété, on peut construire, pour $\epsilon$ assez petit, une partition de l'unité $\left(t_{j}\right)_{1 \leq j \leq q}$ sur $\mathbb{R}^{2 n}$ (i.e $\sum t_{j}=1$ ) telle que, pour tout $j, 0 \leq t_{j} \leq 1, t_{j} \in C^{\infty}\left(\mathbb{R}^{2 n}\right)$ et

$-t_{j}=1 \operatorname{sur}\left\{(x, \xi) ;|x| \leq R=\cap p_{j}^{-1}(] \lambda_{j}-\epsilon ; \lambda_{j}+\epsilon[)\right.$,

$-t_{j}=0 \operatorname{sur}\{(x, \xi) ;|x| \leq R,\} \cap p_{k}^{-1}(] \lambda_{k}-\epsilon ; \lambda_{k}+\epsilon[)$ pour tout $k \neq j$.

On pose $a=\sum t_{j} a_{j}$ et on vérifie que cette fonction convient. Sur $p_{j}^{-1}(] \lambda_{j}-\epsilon ; \lambda_{j}+\epsilon[)$,

$$
\left\{p_{j}, a\right\}=\sum_{k=1}^{q}\left\{p_{j}, C_{k} t_{k} \chi_{k} f_{k}\right\}+\left\{p_{j}, x \cdot \xi\right\} .
$$

Si $|x| \leq R "$ alors

$$
\left\{p_{j}, a\right\}=\left\{p_{j}, C_{j} t_{j} \chi_{j} f_{j}\right\}+\left\{p_{j}, x \cdot \xi\right\}=\left\{p_{j}, a_{j}\right\} \geq C_{0} .
$$

Si $|x|>R$ " alors

$$
\left\{p_{j}, a\right\}=\left\{p_{j}, x \cdot \xi\right\} \geq \lambda_{j} / 2
$$

car $R^{\prime \prime}>R^{\prime}$ et pour $|x|>R^{\prime}, x \notin \operatorname{supp}_{k}$, pour tout $k$.

Poursuivons la preuve de la Proposition 12. Grâce aux hypothèses de "non-croisement" (cf. Définition 2) et de stabilité semi-classique (cf. Définition 1), pour tout $j$,

$$
l \neq l^{\prime} \Longrightarrow\left(\forall x \in \mathbb{R}^{n}, \lambda_{j l}(x ; 0)-E_{j}-\left(E-E_{j}\right) \neq \lambda_{j l^{\prime}}(x ; 0)-E_{j}-\left(E-E_{j}\right)\right),
$$




$$
j \neq j^{\prime} \Longrightarrow\left(\forall x \in \mathbb{R}^{n}, \forall l, l^{\prime}, \lambda_{j l}(x ; 0)-E_{j}-\left(E-E_{j}\right) \neq \lambda_{j^{\prime} l^{\prime}}(x ; 0)-E_{j^{\prime}}-\left(E-E_{j^{\prime}}\right)\right) .
$$

On peut donc appliquer le Lemme 13 aux hamiltoniens classiques $p_{j l}(x, \xi)-E_{j}=|\xi|^{2}+$ $\lambda_{j l}(x ; 0)-E_{j}$ aux énergies $E-E_{j}$. Soit $a$ la fonction "multi-fuite" globale correspondante. D'après (8) et (9), on peut choisir le $R$ de la preuve de ce Lemme 13 de sorte que,

$$
\left\|\Pi_{j}(x ; h) x \cdot\left(\nabla_{x} I_{a}\right)(x ; h) \Pi_{j}(x ; h)\right\|+\left\|2 \Pi_{j}(x ; h)\left(P_{e}(x ; h)-E_{j}\right) \Pi_{j}(x ; h)\right\| \leq E-E_{j},
$$

pour tout $j$ et pour $|x| \geq R$. Soient $\tau_{1}, \tau_{2} \in C^{\infty}\left(\mathbb{R}^{n}\right)$ telles que $\tau_{1}^{2}+\tau_{2}^{2}=1$. On impose que $\tau_{1}$ vaille 1 sur $\left\{x ;|x| \leq R^{\prime}\right\}$ et soit à support dans $\left\{x ;|x| \leq R^{\prime \prime}\right\}$. On pose

$$
F^{A D}=\sum_{j=1}^{r}\left(\sum_{l=1}^{l(j)}\left(\tau_{1} \Pi_{j l} a^{w} \Pi_{j l} \tau_{1}+\tau_{2} \Pi_{j} a^{w} \Pi_{j} \tau_{2}\right)\right),
$$

où $a^{w}$ l'opérateur $h$-pseudo-différentiel de symbole de Weyl $a$. D'après la régularité des projecteurs $\Pi_{j l}$ et le choix du support de $\tau_{1}$ d'une part, les propriétés des $\Pi_{j}$ et celles de a d'autre part, l'opérateur $F^{A D}$ vérifie (1).

On calcule le commutateur $i\left[P^{A D}, F^{A D}\right]$ modulo " $O\left(h^{2}\right)$ ", où " $O\left(h^{2}\right)$ " (respectivement " $O(h)$ ") désignera des opérateurs $B P^{A D}$-bornés tels que la norme de $B\left(P^{A D}+i\right)^{-1}$ soit un $O\left(h^{2}\right)$ (respectivement $O(h)$ ). D'une part, pour tout $j$,

$$
\begin{aligned}
i\left[P^{A D}, \tau_{2} \Pi_{j} a^{w} \Pi_{j} \tau_{2}\right]= & \tau_{2} \Pi_{j} i\left[P, a^{w}\right] \Pi_{j} \tau_{2}+\Pi i\left[-h^{2} \Delta_{x}, \tau_{2} \Pi_{j}\right] a^{w} \Pi_{j} \tau_{2}+\tau_{2} \Pi_{j} a^{w} i\left[-h^{2} \Delta_{x}, \tau_{2} \Pi_{j}\right] \Pi \\
= & \tau_{2} \Pi_{j} i\left[P, a^{w}\right] \Pi_{j} \tau_{2}+" O\left(h^{2}\right)^{"} \\
& -2 i \Pi h \nabla_{x} \cdot h\left(\nabla_{x}\left(\tau_{2} \Pi_{j}\right)\right) a^{w} \Pi_{j} \tau_{2}-2 i \tau_{2} \Pi_{j} a^{w} h\left(\nabla_{x}\left(\tau_{2} \Pi_{j}\right)\right) \cdot h \nabla_{x} \Pi .
\end{aligned}
$$

La somme, pour $1 \leq j \leq r$, des termes faisant intervenir $\left(\nabla_{x} \Pi_{j}\right)$ forme un opérateur $h$-pseudo-différentiel de symbole principal

$$
-2 \tau_{2}^{2}(x) a(x, \xi) \xi \cdot \sum_{j=1}^{r} \Pi(x)\left(\left(\nabla_{x} \Pi_{j}\right)(x) \Pi_{j}(x)+\Pi_{j}(x)\left(\nabla_{x} \Pi_{j}\right)(x)\right) \Pi(x),
$$

qui est nul car

$$
\begin{aligned}
\sum_{j=1}^{r} \Pi(x)\left(\left(\nabla_{x} \Pi_{j}\right)(x) \Pi_{j}(x)+\Pi_{j}(x)\left(\nabla_{x} \Pi_{j}\right)(x)\right) \Pi(x) & =\sum_{j=1}^{r} \Pi(x)\left(\nabla_{x} \Pi_{j}\right)(x) \Pi(x) \\
& =\Pi(x)\left(\nabla_{x} \Pi\right)(x) \Pi(x)=0,
\end{aligned}
$$

d'après la Remarque 10. Comme les commutateurs $\left[\Pi_{j}, a^{w}\right],\left[\Pi_{j}, h \nabla_{x}\right]$ sont des " $O(h)$ ",

$$
\begin{aligned}
\sum_{j=1}^{r} i\left[P^{A D}, \tau_{2} \Pi_{j} a^{w} \Pi_{j} \tau_{2}\right]= & \sum_{j=1}^{r} \tau_{2} \Pi_{j} i\left[P, a^{w}\right] \Pi_{j} \tau_{2}+" O\left(h^{2}\right) " \\
& -2 i \sum_{j=1}^{r}\left(\Pi h \nabla_{x} \cdot\left(h \nabla \tau_{2}\right) \Pi_{j} a^{w} \Pi_{j} \tau_{2}+\tau_{2} \Pi_{j} a^{w} \Pi_{j}\left(h \nabla \tau_{2}\right) \cdot h \nabla_{x} \Pi\right) \\
= & \sum_{j=1}^{r} \tau_{2} \Pi_{j} i\left[P, a^{w}\right] \Pi_{j} \tau_{2}+" O\left(h^{2}\right) " \\
& -2 i \sum_{j=1}^{r}\left(\Pi h \nabla_{x} \cdot\left(h \nabla \tau_{2}\right) \tau_{2} \Pi_{j} a^{w} \Pi_{j}+\Pi_{j} a^{w} \Pi_{j} \tau_{2}\left(h \nabla \tau_{2}\right) \cdot h \nabla_{x} \Pi\right)
\end{aligned}
$$


car le commutateur $\left[\tau_{2}, a^{w}\right]$ est " $O(h)$ ". Comme $\tau_{2}$ est nulle au voisinage du support de $\chi_{j}$ (cf. Lemme 13), pour tout $j$, on peut en fait remplacer $a^{w}$ par $O p_{h}^{w}(x \cdot \xi)$, l'opérateur $h$-pseudo-différentiel de symbole de Weyl $x \cdot \xi$. D'autre part, on peut écrire

$$
\begin{aligned}
\sum_{j=1}^{r}\left(\sum_{l=1}^{l(j)} i\left[P^{A D}, \tau_{1} \Pi_{j l} a^{w} \Pi_{j l} \tau_{1}\right]\right)= & \sum_{j=1}^{r}\left(\sum_{l=1}^{l(j)} \tau_{1} \Pi_{j l} i\left[P, a^{w}\right] \Pi_{j l} \tau_{1}\right)+" O\left(h^{2}\right) "+ \\
& \sum_{j=1}^{r}\left(\sum _ { l = 1 } ^ { l ( j ) } \left(-2 i \Pi h \nabla_{x} \cdot h\left(\nabla_{x}\left(\tau_{1} \Pi_{j l}\right)\right) a^{w} \Pi_{j l} \tau_{1}\right.\right. \\
& \left.\left.-2 i \tau_{1} \Pi_{j l} a^{w} h\left(\nabla_{x}\left(\tau_{1} \Pi_{j l}\right)\right) \cdot h \nabla_{x} \Pi\right)\right) .
\end{aligned}
$$

Les termes contenant les $\left(\nabla_{x} \Pi_{j l}\right)$ forment un opérateur $h$-pseudo-différentiel admissible de symbole principal

$$
\begin{gathered}
-2 i h \sum_{j=1}^{r}\left(\sum _ { l = 1 } ^ { l ( j ) } \left(\tau_{1}(x) \Pi(x) h\left(\nabla_{x} \Pi_{j l}\right)(x) \cdot \xi a(x, \xi) \Pi_{j l}(x) \tau_{1}(x)\right.\right. \\
\left.\left.\quad+\tau_{1}(x) \Pi_{j l}(x) a(x, \xi) \xi \cdot h\left(\nabla_{x} \Pi_{j l}\right)(x) \Pi(x) \tau_{1}(x)\right)\right) .
\end{gathered}
$$

Comme, d'après (7),

$$
\begin{aligned}
& \sum_{j=1}^{r} \Pi_{j}\left(\sum_{l=1}^{l(j)}\left(\left(\nabla_{x} \Pi_{j l}\right) \Pi_{j l}+\Pi_{j l}\left(\nabla_{x} \Pi_{j l}\right)\right)\right) \Pi_{j}=\sum_{j=1}^{r} \Pi\left(\nabla_{x} \Pi_{j}\right) \Pi=0, \\
& \sum_{j=1}^{r}\left(\sum_{l=1}^{l(j)} i\left[P^{A D}, \tau_{1} \Pi_{j l} a^{w} \Pi_{j l} \tau_{1}\right]\right)= \sum_{j=1}^{r}\left(\sum_{l=1}^{l(j)} \tau_{1} \Pi_{j l} i\left[P, a^{w}\right] \Pi_{j l} \tau_{1}\right)+" O\left(h^{2}\right)^{\prime}+ \\
& \sum_{j=1}^{r}\left(\sum _ { l = 1 } ^ { l ( j ) } \left(-2 i \Pi h \nabla_{x} \cdot\left(h \nabla \tau_{1}\right) \Pi_{j l} a^{w} \Pi_{j l} \tau_{1}\right.\right. \\
&\left.\left.-2 i \tau_{1} \Pi_{j l} a^{w} \Pi_{j l}\left(h \nabla \tau_{1}\right) \cdot h \nabla_{x} \Pi\right)\right) .
\end{aligned}
$$

En utilisant le fait que $\left(\nabla \tau_{1}\right)\left[a^{w}, \Pi_{j l}\right] \tau_{1}=" O(h) "$

$$
\left(\nabla \tau_{1}\right) \Pi_{j} a^{w} \Pi_{j} \tau_{1}=\left(\nabla \tau_{1}\right) \sum_{l, k} \Pi_{j l} a^{w} \Pi_{j k} \tau_{1}=\left(\nabla \tau_{1}\right) \sum_{l} \Pi_{j l} a^{w} \Pi_{j l} \tau_{1}+" O(h)^{\prime},
$$


pour tout $j$. Une relation similaire existe pour $\tau_{1} \Pi_{j} a^{w} \Pi_{j}\left(\nabla \tau_{1}\right)$. On en déduit que

$$
\begin{aligned}
\sum_{j=1}^{r}\left(\sum_{l=1}^{l(j)} i\left[P^{A D}, \tau_{1} \Pi_{j l} a^{w} \Pi_{j l} \tau_{1}\right]\right)= & \sum_{j=1}^{r}\left(\sum_{l=1}^{l(j)} \tau_{1} \Pi_{j l} i\left[P, a^{w}\right] \Pi_{j l} \tau_{1}\right)+" O\left(h^{2}\right)^{\prime} \\
& -2 i \sum_{j=1}^{r}\left(\Pi h \nabla_{x} \cdot\left(h \nabla \tau_{1}\right) \Pi_{j} a^{w} \Pi_{j} \tau_{1}+\tau_{1} \Pi_{j} a^{w} \Pi_{j}\left(h \nabla \tau_{1}\right) \cdot h \nabla_{x} \Pi\right) \\
= & \sum_{j=1}^{r}\left(\sum_{l=1}^{l(j)} \tau_{1} \Pi_{j l} i\left[P, a^{w}\right] \Pi_{j l} \tau_{1}\right)+" O\left(h^{2}\right) " \\
& -2 i \sum_{j=1}^{r}\left(\Pi_{j} h \nabla_{x} \cdot\left(h \nabla \tau_{1}\right) \tau_{1} \Pi_{j} a^{w} \Pi_{j}+\Pi_{j} a^{w} \Pi_{j} \tau_{1}\left(h \nabla \tau_{1}\right) \cdot h \nabla_{x} \Pi\right.
\end{aligned}
$$

car le commutateur $\left[\tau_{1}, a^{w}\right]$ est un "O(h)". En regroupant les deux calculs précédents et en utilisant l'égalité $\tau_{1} \nabla \tau_{1}+\tau_{2} \nabla \tau_{2}=0$, on trouve donc

$$
i\left[P^{A D}, F^{A D}\right]=\sum_{j=1}^{r}\left(\sum_{l=1}^{l(j)} \tau_{1} \Pi_{j l} i\left[P, a^{w}\right] \Pi_{j l} \tau_{1}+\tau_{2} \Pi_{j} i\left[P, O p_{h}^{w}(x \cdot \xi)\right] \Pi_{j} \tau_{2}\right)+" O\left(h^{2}\right) " .
$$

Soit $\eta>0$ assez petit tel que $\left.0, E_{j} \notin\right] E-\eta ; E+\eta\left[\right.$, pour tout $j$, et $\theta \in C^{\infty}(\mathbb{R} ; \mathbb{R})$ valant 1 près de $E$ et à support dans $] E-\eta ; E+\eta[$. Pour tout $j$,

$$
P^{A D} \tau_{2} \Pi_{j}-\tau_{2} \Pi_{j} P_{j}^{A D}=\Pi\left[-h^{2} \Delta_{x}, \tau_{2} \Pi_{j}\right] \Pi_{j}=" O(h) " .
$$

Grâce au calcul fonctionnel d'Helffer-Sjöstand (cf. [HS]),

$$
\theta\left(P^{A D}\right) \tau_{2} \Pi_{j}-\tau_{2} \Pi_{j} \theta\left(P_{j}^{A D}\right)=O(h)
$$

Comme, pour tous $j, l$ et pour $P_{j l}=-h^{2} \Delta_{x}+\lambda_{j l}(x ; 0)$,

$$
\begin{aligned}
& P^{A D} \tau_{1} \Pi_{j l}-\tau_{1} \Pi_{j l} P_{j l}= \Pi\left(P_{e}(\cdot ; h)-P_{e}(\cdot ; 0)\right) \tau_{1} \Pi_{j l}+\Pi\left[-h^{2} \Delta_{x}, \tau_{1} \Pi_{j l}\right] \\
&= \Pi(\cdot ; h)\left(P_{e}(\cdot ; h) \Pi_{j l}(\cdot ; h)-P_{e}(\cdot ; 0) \Pi_{j l}(\cdot ; 0)\right) \tau_{1} \\
&-\Pi(\cdot ; h) P_{e}(\cdot ; 0)\left(\Pi_{j l}(\cdot ; h)-\Pi_{j l}(\cdot ; 0)\right) \tau_{1}+" O(h) " \\
&= " O(h) ", \\
& \theta\left(P^{A D}\right) \tau_{1} \Pi_{j l}-\tau_{1} \Pi_{j l} \theta\left(P_{j l}\right)=O(h) .
\end{aligned}
$$

par ce même calcul fonctionnel, et

$$
\begin{aligned}
\theta\left(P^{A D}\right) \tau_{1} \Pi_{j l} i\left[P, a^{w}\right] \Pi_{j l} \tau_{1} \theta\left(P^{A D}\right) & =\theta\left(P^{A D}\right) \tau_{1} \Pi_{j l} i\left[P_{j l}, a^{w}\right] \Pi_{j l} \tau_{1} \theta\left(P^{A D}\right) \\
& =\tau_{1} \Pi_{j l} \theta\left(P_{j l}\right) i\left[P_{j l}, a^{w}\right] \theta\left(P_{j l}\right) \Pi_{j l} \tau_{1}+O\left(h^{2}\right) \\
& \geq c h \tau_{1} \Pi_{j l} \theta^{2}\left(P_{j l}\right) \Pi_{j l} \tau_{1}+O\left(h^{2}\right)
\end{aligned}
$$

pour un certain $c>0$ car $a$ est une fonction fuite globale pour $p_{j}-E_{j}$ à l'énergie $E-E_{j}$. En utilisant de nouveau (10), ce terme est donc minoré par

$$
\operatorname{ch} \theta\left(P^{A D}\right) \tau_{1} \Pi_{j l} \tau_{1} \theta\left(P^{A D}\right)+O\left(h^{2}\right),
$$


pour tout $j$ et tout $l$. D'autre part, pour tout $j$,

$$
\begin{gathered}
\theta\left(P^{A D}\right) \tau_{2} \Pi_{j} i\left[P, O p_{h}^{w}(x \cdot \xi)\right] \Pi_{j} \tau_{2} \theta\left(P^{A D}\right)=h \theta\left(P^{A D}\right) \tau_{2} \Pi_{j}\left(-2 h^{2} \Delta_{x}-x \cdot\left(\nabla_{x} I_{a}\right)\right) \Pi_{j} \tau_{2} \theta\left(P^{A D}\right) \\
=h \tau_{2} \theta\left(P_{j}^{A D}\right) \Pi_{j}\left(2\left(P_{j}^{A D}-E_{j}\right)-x \cdot\left(\nabla_{x} I_{a}\right)-2\left(P_{e}-E_{j}\right)\right) \Pi_{j} \theta\left(P_{j}^{A D}\right) \tau_{2}+O\left(h^{2}\right) \\
\geq h \tau_{2} \Pi_{j} \theta\left(P_{j}^{A D}\right)\left(3\left(E-E_{j}\right) / 2-x \cdot\left(\nabla_{x} I_{a}\right)-2\left(P_{e}-E_{j}\right)\right) \theta\left(P_{j}^{A D}\right) \Pi_{j} \tau_{2}+O\left(h^{2}\right) \\
\geq h \theta\left(P^{A D}\right) \tau_{2} \Pi_{j}\left(3\left(E-E_{j}\right) / 2-x \cdot\left(\nabla_{x} I_{a}\right)-2\left(P_{e}-E_{j}\right)\right) \Pi_{j} \tau_{2} \theta\left(P^{A D}\right)+O\left(h^{2}\right) \\
\geq h \frac{E-E_{j}}{2} \theta\left(P^{A D}\right) \tau_{2} \Pi_{j} \tau_{2} \theta\left(P^{A D}\right)+O\left(h^{2}\right)
\end{gathered}
$$

d'après (5) et (9). En regroupant les inégalités, on peut écrire

$$
\begin{aligned}
\theta\left(P^{A D}\right) i\left[P^{A D}, F^{A D}\right] \theta\left(P^{A D}\right) & \geq c^{\prime} h \theta\left(P^{A D}\right)\left[\sum_{j=1}^{r}\left(\sum_{l=1}^{l(j)} \tau_{1} \Pi_{j l} \tau_{1}+\tau_{2} \Pi_{j} \tau_{2}\right)\right] \theta\left(P^{A D}\right)+O\left(h^{2}\right) \\
& \geq c^{\prime} h \theta\left(P^{A D}\right)^{2}+O\left(h^{2}\right)
\end{aligned}
$$

pour un certain $c^{\prime}>0$, ce qui termine la preuve de cette Proposition 12.

Remarque 14. Dans cette preuve, on a utilisé la fonction "multi-fuite", c'est-à-dire une fonction fuite globale commune aux différents hamiltoniens classiques pour affirmer que le symbole principal donné par (8) est nul. Ceci n'est plus clair si l'on prend des fonctions $a_{j l}$ a priori distinctes, chaque $a_{j l}$ étant une fonction fuite globale pour $|\xi|^{2}+\lambda_{j l}(x ; 0)-E_{j}$ à l'énergie $E-E_{j}$, et si l'on pose

$$
F^{A D}=\sum_{j=1}^{r}\left(\sum_{l=1}^{l(j)} \tau_{1} \Pi_{j l} a_{j}^{w} \Pi_{j l} \tau_{1}+\tau_{2} \Pi_{j} O p_{h}^{w}(x \cdot \xi) \Pi_{j} \tau_{2}\right)
$$

(on a noté par $O p_{h}^{w}(x \cdot \xi)$ l'opérateur h-pseudo-différentiel de symbole de Weyl $x \cdot \xi$ ).

Démonstration de du Théorème 4 : A partir de l'estimation de Mourre semi-classique de la Proposition 12, on peut reprendre la méthode de Mourre en suivant le paramètre $h$ et obtenir (1). C'est précisément ce que le Théorème 3.2 de [KMW1] affirme. Sous la condition (2), on utilise le Théorème 3.4 de [KMW1], qui nous donne l'approximation (3) du Théorème 4. Il reste donc à établir l'estimation (4) pour $\rho>0$.

On établit d'abord l'estimation de Mourre. Comme opérateur conjugué pour $P$, on considère l'opérateur $F^{A D}$ utilisé dans la preuve de la Proposition 12, qui est donné par (6). Notons que le double commutateur $\left[\left[P, F^{A D}\right], F^{A D}\right]$ vérifie l'estimation (1). On exploite la condition (2) sur l'énergie $E$ dans le 
Lemme 15. ([KMW1], Lemme 4.3) Soient $\theta \in C_{0}^{\infty}(\mathbb{R} ; \mathbb{R})$ telle que la borne supérieure de son support soit strictement inférieure à $E^{A D}$, i.e.

$$
\sup (\operatorname{supp} \theta)<E^{A D} .
$$

Alors

$$
\left\|\theta(P(h))-\theta\left(P^{A D}(h)\right)\right\|=O(h)
$$

Démonstration : Il suffit de remarquer que la preuve du Lemme 4.3 de [KMW1] est encore valable sous l'hypothèse $\left(D_{\rho}\right)$ avec $\rho>0$.

Soit $\eta>0$ assez petit tel que $\left.0, E_{j} \notin\right] E-\eta ; E+\eta\left[\right.$, pour tout $j$, et $\theta \in C^{\infty}(\mathbb{R} ; \mathbb{R})$ valant 1 près de $E$ et à support dans $] E-\eta ; E+\eta[$. D'après le Lemme 15 ,

$$
\theta(P) i\left[P, F^{A D}\right] \theta(P)=\theta\left(P^{A D}\right) i\left[P, F^{A D}\right] \theta\left(P^{A D}\right)+O\left(h^{2}\right) .
$$

Comme le noyau de $P^{A D}$ est contenu dans l'image de $1-\Pi$ et $\theta(0)=0, \theta\left(P^{A D}\right)=$ $\Pi \theta\left(P^{A D}\right)=\theta\left(P^{A D}\right) \Pi$ et

$$
\theta\left(P^{A D}\right) i\left[P, F^{A D}\right] \theta\left(P^{A D}\right)=\theta\left(P^{A D}\right) i\left[P^{A D}, F^{A D}\right] \theta\left(P^{A D}\right) .
$$

Par conséquent, d'après la preuve de la Proposition 12, on peut écrire

$$
\theta(P) i\left[P, F^{A D}\right] \theta(P) \geq \operatorname{ch} \theta^{2}\left(P^{A D}\right)+O\left(h^{2}\right) \geq \operatorname{ch} \theta^{2}(P)+O\left(h^{2}\right)
$$

en réutilisant le Lemme 15. On obtient ainsi l'estimation de Mourre semi-classique pour $P$. La méthode de Mourre à paramètre donne l'estimation (4).

Remarque 16. Si un croisement de valeurs propres a lieu à une énergie strictement supérieure à $E$ (cf. Remarque 6), le Théorème 4 reste valable. Les arguments précédents doivent être modifiés car les projecteurs $\Pi_{j l}$ peuvent présenter des singularités au croisement. Mais comme les propriétés de "fuite" de la fonction a sont localisées sur des surfaces d'énergie qui sont éloignées du croisement, on peut imposer que a s'annule près du croisement. Dans ce cas, l'opérateur $F^{A D}$, défini par (6), est encore un opérateur pseudo-différentiel et on peut reprendre la preuve précédente.

\section{Approximation semi-classiques des opérateurs d'onde de canal.}

Grâce au Théorème 4 précédent, le Théorème 4.1 de [KMW1] permet, pour des potentiels à courte portée, d'affirmer que les opérateurs d'onde

$$
\Omega_{ \pm}^{N A D}(h)=s-\lim _{t \rightarrow \pm \infty} e^{i h^{-1} t P(h)} e^{-i h^{-1} t P^{A D}(h)} E_{a c}\left(P^{A D}(h)\right)
$$


(où $E_{a c}\left(P^{A D}(h)\right)$ désigne la projection sur le sous-espace absolument continu de $P^{A D}(h)$ ) sont semi-classiquement proches de l'identité, ce qui implique que les opérateurs d'ondes

$$
\Omega_{ \pm}^{A D}(h)=s-\lim _{t \rightarrow \pm \infty} e^{i h^{-1} t P^{A D}(h)} e^{-i h^{-1} t P_{a}(h)} \Pi_{0}(h)
$$

constituent une approximation semi-classique, dans une certaine bande d'énergie, des opérateurs d'onde de canal

$$
\Omega_{ \pm}(h)=s-\lim _{t \rightarrow \pm \infty} e^{i h^{-1} t P(h)} e^{-i h^{-1} t P_{a}(h)} \Pi_{0}(h) .
$$

Avec les notations de la partie 3, on a le

Théorème 17. Sous les hypothèses du Théorème 4 avec $\rho>1$ et $E_{r}<E^{A D}$, soit I un intervalle non-captif pour les hamiltoniens classiques $|\xi|^{2}+\lambda_{j l}(x ; 0)$ (cf. Définition 3), $1 \leq j \leq r$ et $1 \leq l \leq l(j)$, tel que

$$
I \subset] E_{r}, E^{A D}[
$$

ou bien tel que, pour un certain $j \leq r$ (avec la convention " $E_{0}=-\infty$ "),

$$
I \subset] E_{j-1}, \inf _{x, l} \lambda_{j l}(x ; 0)[.
$$

Alors, pour toute fonction $\chi \in C_{0}^{\infty}(I)$, il existe une constante $C_{\chi}>0$ telle que

$$
\begin{gathered}
\left\|\left(\Omega_{ \pm}^{N A D}(h)-1\right) \chi\left(P^{A D}(h)\right)\right\| \leq C_{\chi} h \\
\left\|\left(\Omega_{ \pm}(h)-\Omega_{ \pm}^{A D}(h)\right) \chi\left(P_{a}(h)\right)\right\| \leq C_{\chi} h .
\end{gathered}
$$

Remarque 18. Ce théorème généralise, pour des potentiels réguliers, le résultat de [KMW1] où l'opérateur $P^{A D}$ ne prenait en compte qu'une valeur propre simple de $P^{a}(0)$. Dans [KMW2], le résultat de [KMW1] est obtenu en présence de singularités coulombiennes.

Démonstration : La première estimation découle du fait que l'on peut appliquer le Théorème 4.1 de [KMW1] puisque l'on dispose de l'estimation (1) du Théorème 4. Pour obtenir la seconde, rappelons que

$$
\Omega_{ \pm}=\Omega_{ \pm}^{N A D} \Omega_{ \pm}^{A D}
$$

(cf. [KMW1], [Jec1]). Comme dans [KMW1], on peut remarquer que, si $\phi$ est une fonction de $C_{0}^{\infty}(I)$ vérifiant $\phi \chi=\chi$, alors, grâce à la propriété d'intervertion des opérateurs d'onde,

$$
\left(\Omega_{ \pm}-\Omega_{ \pm}^{A D}\right) \chi\left(P_{a}\right)=\left(\Omega_{ \pm}^{N A D}-1\right) \phi\left(P^{A D}\right) \Omega_{ \pm}^{A D} \chi\left(P_{a}\right)
$$

La seconde estimation provient alors de la première. 
Au sujet de cette approximation semi-classique des opérateurs d'onde de canal, on peut être plus précis. Considérons, pour $1 \leq j \leq r$, les opérateurs d'onde

$$
\Omega_{j, \pm}^{A D}=s-\lim _{t \rightarrow \pm \infty} e^{i h^{-1} t P_{j}^{A D}} e^{-i h^{-1} t P_{a}} \Pi_{j 0} .
$$

D'après les arguments de [KMW1], ces opérateurs $\Omega_{j, \pm}^{A D}$ existent et sont complets (voir aussi le paragraphe 2.3 de [Jec1]). Remarquons qu'en général,

$$
\Omega_{ \pm}^{A D} \neq \sum_{j=1}^{r} \Omega_{j, \pm}^{A D}
$$

Certes les opérateurs $P_{j}^{A D}$ commutent deux à deux mais il se trouve qu'en général,

$$
P^{A D} \neq \sum_{j=1}^{r} P_{j}^{A D}
$$

En fait, l'évolution $e^{i h^{-1} t P^{A D}}$ "mélange les niveaux". Cependant, on dispose de la Proposition 19 suivante, qui précise le Théorème 17.

Proposition 19. Soit $\chi \in C_{0}^{\infty}(\mathbb{R})$ dont le support est un intervalle tel que $0, E_{j} \notin$ supp $\chi$, pour tout $j$. Posons

$$
r_{\chi}=\max \left\{1 \leq j \leq r ; E_{j}<\inf (\operatorname{supp} \chi)\right\}
$$

(avec la convention $r_{\chi}=0$ si $E_{1}>\sup (\operatorname{supp} \chi)$ ). Si le support de $\chi$ est non-captif pour les hamiltioniens classiques $|\xi|^{2}+\lambda_{j}(x ; 0)$, pour $1 \leq j \leq r_{\chi}$, alors

$$
\left\|\left(\Omega_{ \pm}^{A D}(h)-\sum_{j=1}^{r_{\chi}} \Omega_{j, \pm}^{A D}(h)\right) \chi\left(P_{a}(h)\right)\right\|=O(h) .
$$

Démonstration : Signalons tout d'abord que, pour $j>r_{\chi}$,

$$
\Pi_{j 0} \chi\left(P_{a}\right)=\Pi_{j 0} \chi\left(-h^{2} \Delta_{x}+P^{a}\right)=0
$$

car pour $h$ assez petit, les valeurs propres de $P^{a}(h)$ qui tendent vers $E_{j}$ sont au-dessus du support de $\chi$. On a donc $\Omega_{j, \pm}^{A D} \chi\left(P_{a}\right)=0$. Pour obtenir le résultat, il suffit d'avoir

$$
\left\|\left(\Omega_{ \pm}^{A D}-\Omega_{j, \pm}^{A D}\right) \chi\left(P_{a}\right)\right\|=O(h) .
$$

pour $1 \leq j \leq r_{\chi}$. Pour un tel $j$, on introduit les opérateurs d'onde

$$
\Omega_{j, \pm}^{N A D}=s-\lim _{t \rightarrow \pm \infty} e^{i h^{-1} t P^{A D}} e^{-i h^{-1} t P_{j}^{A D}} E_{a c}\left(P_{j}^{A D}\right) .
$$

Signalons que $\Pi_{j} E_{a c}\left(P_{j}^{A D}\right)=E_{a c}\left(P_{j}^{A D}\right)$. Comme

$$
\begin{aligned}
\left(P^{A D}-P_{j}^{A D}\right) \Pi_{j} & =\Pi\left(-h^{2} \Delta_{x}\right) \Pi_{j}-\Pi_{j}\left(-h^{2} \Delta_{x}\right) \Pi_{j}=-\sum_{k \neq j} \Pi\left[-h^{2} \Delta_{x}, \Pi_{k}\right] \Pi_{j} \\
& =O\left(h\langle x\rangle^{-\rho-1}\right) \cdot h \nabla_{x} \Pi_{j}+O\left(h^{2}\langle x\rangle^{-\rho-2}\right) \Pi_{j}
\end{aligned}
$$


on voit, par la méthode de Cook, que ces opérateurs d'onde existent. Grâce à la propriété d'intervertion de ces opérateurs d'onde, les estimations (1) découlent des suivantes

$$
\left\|\left(\Omega_{j, \pm}^{N A D}-1\right) \chi\left(P_{j}^{A D}\right)\right\|=O(h) .
$$

Pour obtenir (2), vérifions que l'on peut reprendre la preuve du Théorème 4.1 de [KMW1]. Tout d'abord, pour $1 \leq j \leq r_{\chi}$, on a l'estimation semi-classique de la résolvante de $P_{j}^{A D}$ sur le support de $\chi$ (cf. Théorème 4) grâce aux hypothèses de non-capture et de "noncroisement" faites sur $E_{j}$. D'après la théorie des opérateurs localement lisses (cf. [RS4]), on a donc, pour tout $s>1 / 2$, l'existence d'une constante $C>0$ telle que

$$
\int_{-\infty}^{\infty}\left\|\langle x\rangle^{-s} e^{-i h^{-1} t P_{j}^{A D}} \chi\left(P_{j}^{A D}\right) f\right\|^{2} d t \leq C\|f\|^{2},
$$

pour toute fonction $f \in L^{2}\left(\mathbb{R}_{x, y}^{n(N+1)}\right)$, uniformément pour $h$ assez petit (cf. le Lemme 4.2 de $[\mathrm{KMW} 1])$. En écrivant, avec $Q_{j}^{A D}=\hat{\Pi}_{j} P \hat{\Pi}_{j}, \hat{\Pi}_{j}=\Pi-\Pi_{j}$,

$$
P^{A D}=P_{j}^{A D}+Q_{j}^{A D}+V_{j}
$$

on peut reprendre la preuve du Lemme 4.3 de [KMW1] et obtenir

$$
\left\|\left(\chi\left(P^{A D}\right)-\chi\left(P_{j}^{A D}\right)\right)\langle x\rangle^{\rho}\right\|=O(h),
$$

pour tout $1 \leq j \leq r_{\chi}$. Enfin, comme

$$
\begin{aligned}
V_{j} \Pi_{j} & =\sum_{k \neq j}\left(\Pi_{k} P \Pi_{j}+\Pi_{j} P \Pi_{k}\right) \Pi_{j} \\
& =\sum_{k \neq j} \Pi_{k}\left(-h^{2} \Delta_{x}\right) \Pi_{j}=-\sum_{k \neq j}\left[-h^{2} \Delta_{x}, \Pi_{k}\right] \Pi_{j} \\
& =O\left(h\langle x\rangle^{-\rho-1}\right) \cdot h \nabla_{x} \Pi_{j}+O\left(h^{2}\langle x\rangle^{-\rho-2}\right) \Pi_{j},
\end{aligned}
$$

les arguments de la preuve de ce Théorème 4.1 de [KMW1] permettent d'obtenir (2).

\section{Autres utilisations d'une fonction fuite ou "multi- fuite" globale.}

La construction d'une fonction fuite ou "multi-fuite" globale permet, dans d'autres situations, d'obtenir des théorèmes d'absorption limite semi-classiques. On va établir de tels théorèmes pour un opérateur de Schrödinger matriciel et, comme dans [Ce], pour l'opérateur de Dirac avec champ électrique scalaire.

Commençons par un cas très similaire à celui traité dans la partie 3. Prenons un opérateur de Schrödinger matriciel de la forme

$$
H=-h^{2} \Delta_{x} I_{m}+M(x),
$$


agissant dans $\left(L^{2}\left(\mathbb{R}^{n}\right)\right)^{m}$, où $I_{m}$ est la matrice identité à $m$ lignes et $m$ colonnes (avec $m>1)$ et $M(x)$ est une matrice symétrique réelle de même taille, vérifiant les propriétés - $\mathbb{R}^{n} \ni x \mapsto M(x)$ est de classe $C^{\infty}$,

- il existe une matrice symétrique réelle $M_{0}$ et un réel $\rho>0$ tels que

$$
\forall \alpha \in \mathbb{N}^{n}, \exists C_{\alpha}>0 ; \forall x \in \mathbb{R}^{n},\left\|\partial_{x}^{\alpha}\left(M(x)-M_{0}\right)\right\| \leq C_{\alpha}\langle x\rangle^{-\rho-|\alpha|} .
$$

Soient $E_{1}<\ldots<E_{r}$ les valeurs propres de $M_{0}$, chaque $E_{j}$ étant de multiplicité $m_{j}$ (on a donc $m=\sum m_{j}$ ). D'après la propriété (1) pour $\alpha=0$, il existe, pour tout $j, m_{j}$ fonctions $\mathbb{R}^{n} \ni x \mapsto \lambda_{j l}(x) \in \sigma(M(x))$ qui tendent vers $E_{j}$ à l'infini. On fait l'hypothèse de "non-croisement"

$$
(j, l) \neq\left(j^{\prime}, l^{\prime}\right) \Longrightarrow\left(\forall x \in \mathbb{R}^{n}, \lambda_{j l}(x) \neq \lambda_{j^{\prime} l^{\prime}}(x)\right) .
$$

En particulier, pour tout $j$, il y a $l(j)$ fonctions $\lambda_{j l}$ qui tendent vers $E_{j}$ à l'infini et chaque $\lambda_{j l}$ est de multiplicité constante. Sous cette hypothèse (2), on peut trouver, pour tout $j$, une famille $\left\{\Gamma_{j}(x), x \in \mathbb{R}^{n}\right\}$ de contours dans $\mathbb{C}$ telle que

$$
\Pi_{j}(x)=\frac{1}{2 i \pi} \int_{\Gamma_{j}(x)}(z-M(x))^{-1} d z
$$

soit le projecteur spectral associée à $\left\{\lambda_{j l}(x), 1 \leq l \leq l(j)\right\}$ et, pour tout $1 \leq l \leq l(j)$, une famille $\left\{\Gamma_{j l}(x), x \in \mathbb{R}^{n}\right\}$ de contours dans $\mathbb{C}$ telle que

$$
\Pi_{j l}(x)=\frac{1}{2 i \pi} \int_{\Gamma_{j l}(x)}(z-M(x))^{-1} d z
$$

soit le projecteur spectral associée à $\lambda_{j l}(x)$. Notons que, pour $|x|$ assez grand, on peut imposer $\Gamma_{j}(x)=\Gamma_{j}$, un contour entourant $E_{j}$ mais aucune des $E_{k}$ pour $k \neq j$. Le projecteur spectral de $M_{0}$ associé à la valeur propre $E_{j}$ est donné par

$$
\Pi_{j 0}=\frac{1}{2 i \pi} \int_{\Gamma_{j}}\left(z-M_{0}\right)^{-1} d z
$$

Grâce à la propriété (1) et à l'hypothèse de "non-croisement" (2), on voit que les projecteurs $\Pi_{j}$ et $\Pi_{j l}$ sont de classe $C^{\infty}$ et, pour tout $\alpha \in \mathbb{N}^{n}$, il existe $D_{\alpha}>0$, tel que

$$
\forall x \in \mathbb{R}^{n},\left\|\partial_{x}^{\alpha}\left(\Pi_{j}(x)-\Pi_{j 0}\right)\right\| \leq D_{\alpha}\langle x\rangle^{-\rho-|\alpha|} .
$$

Notons que, comme dans la partie 3, le contrôle des dérivées des $\Pi_{j l}$ n'est pas clair. En revanche, on voit que les valeurs propres $\lambda_{j l}(x)$ vérifient la condition $\left(D_{\rho}^{\prime}\right)$, introduite dans cette partie 3. Pour obtenir le Théorème 22, on montre la

Proposition 20. On suppose que la fonction $\mathbb{R}^{n} \ni x \mapsto M(x)$ vérifie les propriétés précédentes (cf. (1)) et l'hypothèse de "non-croisement" (2). Soit $E \notin\{0\} \cup\left\{E_{j}, 1 \leq j \leq\right.$ $r\}$ une énergie non-captive pour chaque hamiltonien classique $|\xi|^{2}+\lambda_{j l}(x), 1 \leq j \leq r$ et $1 \leq l \leq l(j)$ (cf. Définition 3). 
Pour $h$ assez petit, il existe un opérateur conjugué F satisfaisant

$$
\left\|[[H, F], F](H+i)^{-1}\right\|=O\left(h^{2}\right)
$$

et vérifiant l'estimation de Mourre

$$
\chi(H) i\left[H, F^{A D}(h)\right] \chi(H) \geq \alpha h \chi^{2}(H)
$$

avec $\chi=\mathbb{1}_{E-\delta, E+\delta[}, \delta>0$ et $\alpha>0$ indépendants de $h$.

Démonstration : La preuve est analogue à celle de la preuve de la Proposition 12 en prenant comme opérateur conjugué

$$
F=\sum_{j=1}^{r}\left(\sum_{l=1}^{l(j)} \tau_{1} \Pi_{j l}\left(a^{w} I_{m}\right) \Pi_{j l} \tau_{1}+\tau_{2} \Pi_{j}\left(a^{w} I_{m}\right) \Pi_{j} \tau_{2}\right),
$$

où $a$ est une fonction "multi-fuite" globale, construite avec le Lemme 13, pour les hamiltoniens classiques $|\xi|^{2}+\lambda_{j l}(x), j$ vérifiant $E_{j}<E$, à l'énergie $E-E_{j}$.

Remarque 21. Imaginons un instant que l'on dispose d'un symbole $(x, \xi) \mapsto A(x, \xi)$, ̀̀ valeurs matricielles et qui commute avec $M(x)$, de sorte qu'il existe $c>0$, tel que

$$
\left\{|\xi|^{2} I_{m}+M(x), A(x, \xi)\right\} \geq c
$$

$(\{\cdot, \cdot\}$ désigne le crochet de Poisson, à valeurs matricielles cette fois, et l'inégalité est également matricielle) sur la couche d'énergie

$$
\left\{(x, \xi) \in \mathbb{R}^{2 n} ; \exists E^{\prime} \in\right] E-\delta, E+\delta\left[; \operatorname{det}\left(|\xi|^{2} I_{m}+M(x)-E^{\prime} I_{m}\right)=0\right\} .
$$

Dans ce cas, on peut démontrer la Proposition 20, sans l'hypothèse de "non-croisement", à l'aide de l'opérateur conjugué $A^{w}$, l'opérateur h-pseudo-différentiel de symbole de Weyl $A(x, \xi)$. Tout le problème réside dans la construction d'un tel opérateur.

En l'absence de "croisement", lorsque les valeurs propres $E_{j}$ sont simples, on cherche des symboles scalaires $a_{j}$ avec

$$
A(x, \xi)=\sum_{j=1}^{m} a_{j}(x, \xi) \Pi_{j}(x)
$$

et, d'après la remarque 14 , il vaut mieux prendre $a_{1}=\cdots=a_{m}=a$, où a est une fonction "multi-fuite" globale pour les valeurs propres de $|\xi|^{2} I_{m}+M(x)$.

Grâce à la Proposition 20, on obtient, par la méthode de Mourre, le

Théorème 22. Soit E satisfaisant les hypothèses de la Proposition 20. Pour tout voisinage compact $\Lambda$ de $E$, assez petit, et pour tout $s>1 / 2$,

$$
\left\|\langle x\rangle^{-s}(\lambda \pm i 0-H)^{-1}\langle x\rangle^{-s}\right\|=O\left(h^{-1}\right),
$$

pour $h$ assez petit et uniformément pour $\lambda \in \Lambda$. 
Plaçons-nous maintenant dans une situation vraiment différente. Par définition, l'opérateur de Dirac semi-classique, avec champ électrique scalaire, dans la représentation de Weyl, est l'opérateur matriciel du premier ordre

$$
D=\sum_{j=1}^{3} h \alpha_{j} D_{j}+\alpha_{4}+V I_{4}
$$

agissant dans $L^{2}\left(\mathbb{R}^{3} ; \mathbb{C}^{4}\right)$, où $D_{j}=\frac{1}{i} \frac{\partial}{\partial x_{j}}$ et $V$ est le potentiel électrique, que l'on supposera régulier et à longue portée. Les $\alpha_{j}$ sont les matrices 4-4

$$
\alpha_{4}=\left(\begin{array}{cc}
0 & I_{2} \\
I_{2} & 0
\end{array}\right), \alpha_{j}=\left(\begin{array}{cc}
\sigma_{j} & 0 \\
0 & \sigma_{j}
\end{array}\right), 1 \leq j \leq 3
$$

où $I_{2}$ est la matrice identité $2-2$ et les $\sigma_{j}$ sont les matrices de Pauli

$$
\sigma_{1}=\left(\begin{array}{ll}
0 & 1 \\
1 & 0
\end{array}\right), \sigma_{2}=\left(\begin{array}{cc}
0 & -i \\
i & 0
\end{array}\right), \sigma_{3}=\left(\begin{array}{cc}
1 & 0 \\
0 & -1
\end{array}\right)
$$

(cf. [T], [W2]). Le $h$-symbole de l'opérateur libre (c'est-à-dire pour $V=0$ )

$$
\sum_{j=1}^{3} \alpha_{j} \xi_{j}+\alpha_{4}
$$

qui, pour tout $(x, \xi)$, est une matrice 4 -4, admet deux valeurs propres doubles $\pm\langle\xi\rangle$ avec $\langle\xi\rangle=\sqrt{1+|\xi|^{2}}$. Notons par $\Pi_{ \pm}(\xi)$ le projecteur spectral associé. Ces projecteurs, que l'on peut expliciter à l'aide de l'opérateur de Foldy-Wouthuysen (cf. [T], [W2]), sont réguliers en $\xi$. Le $h$-symbole de l'opérateur $D$,

$$
d(x, \xi)=\sum_{j=1}^{3} \alpha_{j} \xi_{j}+\alpha_{4}+V(x) I_{4},
$$

admet donc lui aussi deux valeurs propres doubles $p_{ \pm}(x, \xi)= \pm\langle\xi\rangle+V(x)$. Essayons d'appliquer la méthode précédente à cet opérateur $D$. Il convient donc de fabriquer une fonction fuite globale pour $p_{+}$et une pour $p_{-}$. Mais, sous les hypothèses faites sur $V$, il n'existe pas de telles fonctions! En effet, pour $\lambda_{0} \in \mathbb{R}$, les surfaces d'énergie $p_{+}^{-1}\left(\lambda_{0}\right)$ et $p_{-}^{-1}\left(\lambda_{0}\right)$ ne sont jamais simultanément non-compactes.

Cependant, il est possible d'obtenir un théorème d'absorption limite semi-classique dans le cas où l'une de ces surfaces est vide. On retrouve ainsi le résultat de [Ce], avec une hypothèse moins restrictive sur $V$.

Démonstration de du Théorème 7 : D'après la minoration (6) imposée à $\lambda_{0}$, on a, pour $\epsilon>0$ assez petit,

$$
] \lambda_{0}-\epsilon ; \lambda_{0}+\epsilon\left[\cap \sigma\left(P_{-}\right)=\emptyset\right.
$$

où $P_{-}=O p_{h}^{w}\left(p_{-}\right)$. La contribution de $P_{-}$dans l'estimation de Mourre disparaîtra. Il suffit donc de construire une fonction fuite globale pour $p_{+}$. C'est l'objet du 
Lemme 23. On suppose que le potentiel $V$ vérifie la condition $\left(D_{\rho}^{\prime}\right)$ pour $\rho>0$. Pour tout énergie $\lambda_{0}>1$, non-captive pour $p_{+}$(cf. Définition 3), il existe une fonction a $\in C^{\infty}\left(\mathbb{R}^{6}\right)$ et des réels $\epsilon>0, C_{0}>0$ tels que, sur $p_{+}^{-1}(] \lambda_{0}-\epsilon ; \lambda_{0}+\epsilon[)$,

$$
\left\{p_{+}, a\right\} \geq C_{0}
$$

Démonstration : On s'inspire encore de [GM]. Sur la couche $p_{+}^{-1}(] \lambda_{0}-\epsilon ; \lambda_{0}+\epsilon[)$,

$$
\begin{aligned}
\left\{p_{+}, x \cdot \xi\right\}(x, \xi) & =\frac{|\xi|^{2}}{\langle\xi\rangle}-x \cdot \nabla V(x) \\
& \geq \lambda_{0}-\epsilon-\frac{1}{\langle\xi\rangle}-x \cdot \nabla V(x)-V(x)
\end{aligned}
$$

D'après $\left(D_{\rho}^{\prime}\right)$, on peut trouver un réel $R>0$ tel que, pour $\epsilon>0$ assez petit,

$$
(x, \xi) \in p_{+}^{-1}(] \lambda_{0}-\epsilon ; \lambda_{0}+\epsilon[) \cap\{(x, \xi) ;|x| \geq R\} \Longrightarrow\left\{p_{+}, x \cdot \xi\right\}(x, \xi) \geq \frac{\lambda_{0}-1}{2}
$$

Soit $x \mapsto g(x)$ une fonction de $C_{0}^{\infty}\left(\left\{y ;|y| \leq R_{1}\right\}\right)$ avec $R<R_{1}$, valant 1 pour $|x| \leq R$ et vérifiant $0 \leq g \leq 1$. On pose

$$
f_{+}(x, \xi)=-\int_{0}^{+\infty} g \circ \phi_{1,+}^{t}(x, \xi) d t
$$

où $\phi_{1,+}^{t}(x, \xi)$ est la composante spatiale du flot $\phi_{+}^{t}(x, \xi)$ associé à $p_{+}$. D'après l'hypothèse de non-capture, sur $p_{+}^{-1}(] \lambda_{0}-\epsilon ; \lambda_{0}+\epsilon[)$, cette fonction $f_{+}$est de classe $C^{\infty}$ et

$$
\left\{p_{+}, f_{+}\right\}(x, \xi)=g(x) .
$$

De plus, elle est uniformément bornée par $T_{R_{1},+}$, le temps de séjour dans la boule $\{x ;|x| \leq$ $\left.R_{1}\right\}$ pour la dynamique associée à $p_{+}$. Soient $\chi_{+} \in C_{0}^{\infty}\left(\mathbb{R}^{n}\right)$ valant 1 sur le support de $g$ et $C_{+}>0$, on pose

$$
a(x, \xi)=x \cdot \xi+C_{+} \chi_{+}(x) f_{+}(x, \xi) .
$$

La fonction $a$ est $C^{\infty} \operatorname{sur} p_{+}^{-1}(] \lambda_{0}-\epsilon ; \lambda_{0}+\epsilon[)$ et

$$
\left\{p_{+}, a\right\}=C_{+} g+C_{+} f_{+}\left\{p_{+}, \chi_{+}\right\}+\left\{p_{+}, x \cdot \xi\right\}
$$

Pour $C_{+}$assez grand et grâce à $(4)$, sur $p_{+}^{-1}(] \lambda_{0}-\epsilon ; \lambda_{0}+\epsilon[)$,

$$
C_{+} g+\left\{p_{+}, x \cdot \xi\right\} \geq \frac{\lambda_{0}-1}{2} .
$$

En choisissant maintenant les variations de $\chi_{+}$assez petites (ce qui est possible en agmentant la taille de son support), on assure, sur $p_{+}^{-1}(] \lambda_{0}-\epsilon ; \lambda_{0}+\epsilon[)$, que

$$
\left|C_{+} f_{+}\left\{p_{+}, \chi_{+}\right\}\right| \leq \frac{\lambda_{0}-1}{4} .
$$


Reprenons la preuve du Théorème 7 . Posons $F=\Pi_{+} a^{w} \Pi_{+}$. Cet opérateur vérifie l'estimation

$$
\left\|[[D, F], F](D+i)^{-1}\right\|=O\left(h^{2}\right) .
$$

On se propose d'obtenir l'estimation de Mourre semi-classique près de $\lambda_{0}$ avec cet opérateur $F$. Soit $\theta \in C^{\infty}(\mathbb{R})$ valant 1 près de $\lambda_{0}$ et à support dans $] \lambda_{0}-\epsilon ; \lambda_{0}+\epsilon[$ (avec le $\epsilon \mathrm{du}$ Lemme 23). En posant $P_{+}=O p_{h}^{w}\left(p_{+}\right)$,

$$
\theta(D)=\theta\left(P_{+}\right) \Pi_{+}+\theta\left(P_{-}\right) \Pi_{-}=\theta\left(P_{+}\right) \Pi_{+} .
$$

d'après (3). Par conséquent, on peut écrire

$$
\begin{aligned}
\theta(D) i[D, F] \theta(D) & =\theta\left(P_{+}\right) \Pi_{+} i[D, F] \Pi_{+} \theta\left(P_{+}\right) \\
& =\theta\left(P_{+}\right) \Pi_{+} i\left[P_{+}, \Pi_{+} a^{w} \Pi_{+}\right] \Pi_{+} \theta(D) .
\end{aligned}
$$

Les opérateurs $\Pi_{+}$et $P_{+}$ne commutent pas forcément mais

$$
\begin{aligned}
\Pi_{+} i\left[P_{+}, \Pi_{+} a^{w} \Pi_{+}\right] \Pi_{+}= & \Pi_{+} i\left[P_{+}, a^{w}\right] \Pi_{+} \\
& +\Pi_{+} a^{w} i\left[V I_{4}, \Pi_{+}\right] \Pi_{+} \\
& +\Pi_{+} i\left[V I_{4}, \Pi_{+}\right] a^{w} \Pi_{+} .
\end{aligned}
$$

Le $h$-symbole principal de Weyl des deux derniers termes est

$$
h \Pi_{+}(\xi)\left\{V, \Pi_{+}\right\}(x, \xi) a(x, \xi) \Pi_{+}(\xi)=-h a(x, \xi)(\nabla V)(x) \cdot \Pi_{+}(\xi)\left(\nabla_{\xi} \Pi_{+}\right)(\xi) \Pi_{+}(\xi)=0
$$

d'après la Remarque 10. On en déduit que

$$
\begin{aligned}
\theta(D) i[D, F] \theta(D) & =\theta\left(P_{+}\right) \Pi_{+} a^{w} i\left[P_{+}, a^{w}\right] \Pi_{+} \theta\left(P_{+}\right)+O\left(h^{2}\right) \\
& \geq \operatorname{ch} \theta\left(P_{+}\right)^{2} \Pi_{+}+O\left(h^{2}\right) \\
& \geq \operatorname{ch} \theta(D)^{2}+O\left(h^{2}\right)
\end{aligned}
$$

pour un certain $c>0$, d'après (5). De nouveau, on termine la preuve par la méthode de Mourre à paramètre.

\section{Références}

[A] S.Agmon : Lectures on Exponential Decay of Solutions of Second-Order Elliptic Equations. Princeton University Press, 1982.

[BO] M.Born, R.Oppenheimer : Zur Quantentheorie der Molekeln. Annalen der Physik, 84, 457, 1927.

[Ce] S.Cerbah : Principe d'absoption limite semi-classique pour l'opérateur de Dirac. preprint.

[CDS] J.M.Combes, P.Duclos, R.Seiler : The Born-Oppenheimer Approximation. Rigourous Atomic and Molecular Physics, eds. Wightman and Velo, Plenum, New York, 1981. 
[CT] J.M.Combes, A.Tip : Properties of the scattering amplitude for electron-atom collisions. Ann. I.H.P., vol. 40, $n^{\circ}$ 2, 1984, p. 117-139.

[GM] C.Gérard, A.Martinez : Principe d'absorption limite pour des opérateurs de Schrödinger à longue portée. C.R. Acad. Sci. 306, 121-123, 1988.

[H] G.A.Hagedorn : Molecular propagation through electron energy level crossings. Memoirs AMS 536, vol. 111, 1994.

[HS] B.Helffer, J.Sjöstrand : Opérateurs de Schrödinger avec champs magnétiques faibles et constants. Exposé No. XII, Séminaire EDP, février 1989, Ecole Polytechnique.

[Jec1] Th.Jecko : Sections efficaces totales d'une molécule diatomique dans l'approximation de Born-Oppenheimer. Thèse de doctorat, université de Nantes, 1996.

[Jec2] Th.Jecko: Approximation de Born-Oppenheimer de sections efficaces totales diatomiques. preprint.

[JMP] A.Jensen, E.Mourre, P.Perry : Multiple commutator estimates and resolvant smoothness in quantum scattering theory. Ann. IHP 41, 207-225, 1984.

[K] T.Kato : Perturbation Theory for Linear Operators. Springer, Berlin, 1976.

[KMSW] M.Klein, A.Martinez, R.Seiler, X.P.Wang: On the Born-Oppenheimer Expansion for Polyatomic Molecules. Commun. Math. Phys. 143, 606-639, 1992.

[KMW1] M.Klein, A.Martinez, X.P.Wang : On the Born-Oppenheimer Approximation of Wave Operators in Molecular Scattering Theory. Commun. Math. Phys. 152, 73-95, 1993.

[KMW2] M.Klein, A.Martinez, X.P.Wang : On the Born-Oppenheimer Aproximation of Wave Operators II : Singular Potentials. J. of Math. Phys., vol 38, 3, march 97.

[Mo] E.Mourre : Absence of singular continuous spectrum for certain self-adjoint operators. Commun. in Math. Phys. 78, 391-408, 1981.

[Ne] L.Nédélec : Résonances pour l'opérateur de Schrödinger matriciel. Ann.IHP vol 65, 2, 1996, 129-162.

[No] J.Nourrigat: Amplitude de diffusion pour l'opérateur de Dirac. Exposé aux journées semi-classiques à Lille, 22-24 janvier 1997.

[PSS] P.Perry, I.M.Sigal, B.Simon : Spectral analysis of $N$-body Schrödinger operators. Ann. of Math. 114 (1981), 519-567.

[Ra] A.Raphaelian : Ion-Atom Scattering within a Born-Oppenheimer Framework. Disertation TU Berlin, 1986.

[RS3] M.Reed, B.Simon : Methods of Modern Mathematical Physics, Tome III : Scattering Theory. Academic Press 1979.

[RS4] M.Reed, B.Simon : Methods of Modern Mathematical Physics, Tome IV : Analysis of Operators. Academic Press.

[RT] D.Robert, H.Tamura : Semiclassical estimates for resolvents and asymptotics for total cross-section. Ann. IHP 46, 415-442, 1987.

[T] B.Thaller : The Dirac Equation. Springer Verlag 1992. 
[W1] X.P.Wang : Semiclassical Resolvent Estimates for N-body Schrödinger Operators. J. Funct. Anal. 97, 466-483, (1991).

[W2] X.P.Wang : Puits multiples pour l'opérateur de Dirac. Ann. IHP 43, 269-319, (1985). 